\title{
Search for a charged Higgs boson decaying to charm and bottom quarks in proton-proton collisions at $\sqrt{s}=8 \mathrm{TeV}$
}

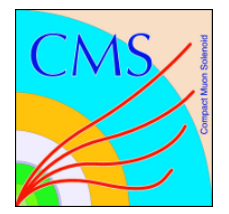

\section{The CMS collaboration}

\section{E-mail: cms-publication-committee-chair@cern.ch}

ABSTRACT: A search for charged Higgs boson decaying to a charm and a bottom quark $\left(\mathrm{H}^{+} \rightarrow \mathrm{c} \overline{\mathrm{b}}\right)$ is performed using $19.7 \mathrm{fb}^{-1}$ of pp collision data at $\sqrt{s}=8 \mathrm{TeV}$. The production mechanism investigated in this search is $t \bar{t}$ pair production in which one top quark decays to a charged Higgs boson and a bottom quark and the other decays to a charged lepton, a neutrino, and a bottom quark. Charged Higgs boson decays to $c \bar{b}$ are searched for, resulting in a final state containing at least four jets, a charged lepton (muon or electron), and missing transverse momentum. A kinematic fit is performed to identify the pair of jets least likely to be the bottom quarks originating from direct top quark decays and the invariant mass of this pair is used as the final observable in the search. No evidence for the presence of a charged Higgs boson is observed and upper limits at 95\% confidence level of $0.8-0.5 \%$ are set on the branching fraction $\mathcal{B}\left(\mathrm{t} \rightarrow \mathrm{H}^{+} \mathrm{b}\right)$, assuming $\mathcal{B}\left(\mathrm{H}^{+} \rightarrow \mathrm{cb}\right)=1.0$ and $\mathcal{B}\left(\mathrm{t} \rightarrow \mathrm{H}^{+} \mathrm{b}\right)+\mathcal{B}(\mathrm{t} \rightarrow \mathrm{Wb})=1.0$, for the charged Higgs boson mass range $90-150 \mathrm{GeV}$.

KEYwORDS: Hadron-Hadron scattering (experiments), Higgs physics

ARXIV EPRINT: 1808.06575 


\section{Contents}

1 Introduction 1

2 Event simulation and reconstruction with CMS detector 2

3 Event selection and yields $\quad 4$

4 Reconstruction of $t \bar{t}$ events 5

$\begin{array}{lll}5 & \text { Systematic uncertainties } & 7\end{array}$

6 Results 10

$\begin{array}{lll}7 & \text { Summary } & 11\end{array}$

$\begin{array}{ll}\text { The CMS collaboration } & 19\end{array}$

\section{Introduction}

In 2012, a boson with a mass about $125 \mathrm{GeV}$ was discovered at the CERN LHC [1-3] with its properties subsequently shown [4-7] to be consistent with those of the standard model (SM) [8-10] Higgs boson [11-16]. Although the last missing particle of the SM has been discovered, several questions remain, including the nature of dark matter $[17,18]$, and the origin of neutrino masses [19] inferred from the observation of neutrino oscillations [20]. Several hypotheses beyond the SM have been introduced and tested to answer these questions, and many of them include more than one Higgs doublet. Models with two Higgs doublets, so-called two-Higgs-doublet model (2HDM) [21, 22], result in five Higgs bosons: two charged $\left(\mathrm{H}^{ \pm}\right)$and three neutral $(\mathrm{A}, \mathrm{H}, \mathrm{h})$. In the $2 \mathrm{HDM}$, the Higgs boson discovered at the LHC can be one of the $\mathrm{CP}$-even neutral bosons ( $\mathrm{H}$ or $\mathrm{h}$ ). Unlike the $\mathrm{SM}$, in general 2HDM allows flavour changing neutral current (FCNC) at tree level. To suppress such tree level FCNC, all fermions with the same electric charge are required to couple to one Higgs doublet only $[23,24]$. The $2 \mathrm{HDM}$ is typically categorized into four different types: type-I, type-II, lepton-specific (type-III), and flipped (type-Y, also known as type-IV), depending on the assignment of up/down-type quark and lepton couplings to each Higgs doublet.

We present a search for charged Higgs bosons. Hereafter, we refer to them as $\mathrm{H}^{+}$, but charge conjugate states are always implied. In the 2HDM, the mass of the charged Higgs boson $\left(M_{\mathrm{H}^{+}}\right)$is an unconstrained parameter. Regardless of its mass, $\mathrm{H}^{+}$is expected to have a large coupling to the top quark unless a specific condition is being considered as in refs. $[25,26]$. If $M_{\mathrm{H}^{+}}$is smaller than the top quark mass, the so-called light charged Higgs boson scenario, the top quark can decay to a $\mathrm{H}^{+}$and a $\mathrm{b}$ quark, $\mathrm{t} \rightarrow \mathrm{H}^{+} \mathrm{b}$. The 

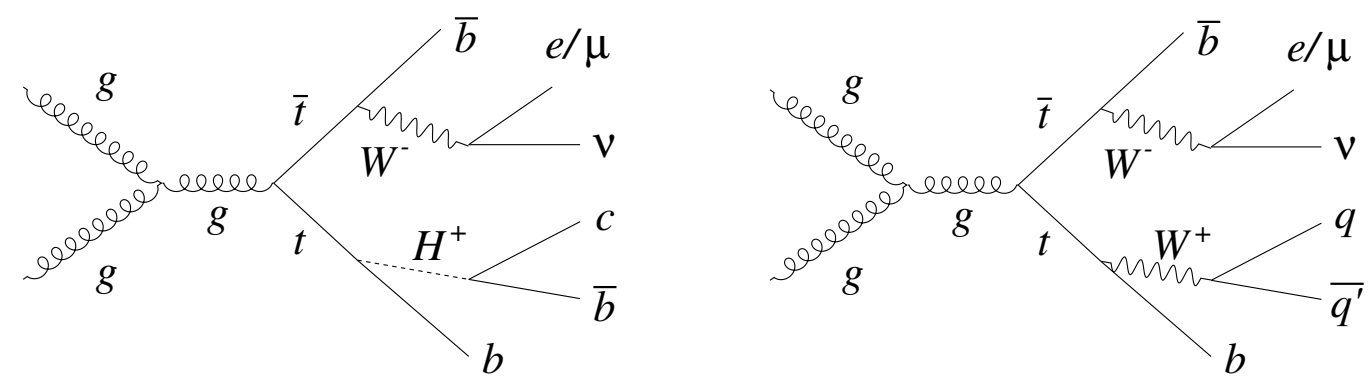

Figure 1. Feynman diagrams of the $\mathrm{H}^{+}$production in top quark pair events (left) compared to the standard model production of $t \bar{t}$ in lepton+jets final states (right).

LEP experiments [27] excluded the mass of charged Higgs below 80 (72.5) GeV for type-II (type-I for pseudo-scalar masses above $12 \mathrm{GeV}$ ) scenario at $95 \%$ confidence level (CL). In the presence of the $\mathrm{W}$ boson resonance at a mass of $80.4 \mathrm{GeV}$, the light charged Higgs boson search range is typically set between the $\mathrm{W}$ boson mass and the top quark mass. Previous direct searches for a light $\mathrm{H}^{+}$in decays of a top quark have been performed at hadron collider experiments in following channels: $\mathrm{H}^{+} \rightarrow \bar{\tau} \nu$ [28-34], $\mathrm{H}^{+} \rightarrow \mathrm{c} \overline{\mathrm{s}}$ [35-38], and $\mathrm{H}^{+} \rightarrow$ WA [39]. No indication of a $\mathrm{H}^{+}$was observed and the best upper limits on the branching fraction of $\mathrm{t} \rightarrow \mathrm{H}^{+} \mathrm{b}$ were placed at $\mathcal{O}(1 \%)$. The $\mathrm{H}^{+} \rightarrow \mathrm{c} \overline{\mathrm{b}}$ process is the dominant decay channel in the type-Y 2HDM [40-42], and this signal could be a signature of models with more than two Higgs doublets [43, 44]. The search is performed assuming $\mathcal{B}\left(\mathrm{H}^{+} \rightarrow \mathrm{c} \overline{\mathrm{b}}\right)=1.0$ without any other model-dependent assumption.

The search uses t $\bar{t}$ events with a final state of at least four jets (at least two of which originate from b quarks), a charged lepton (muon or electron), and missing transverse

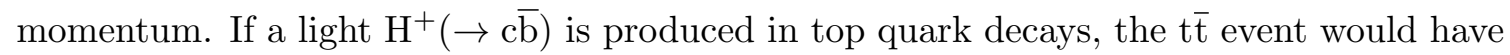
one more jet to be identified originating from $b$ quark due to the $\mathrm{H}^{+}$decays, as shown in figure 1. A kinematic fit is performed to identify the pair of jets least likely to be the $b$ quarks originating from direct top quark decays. The invariant mass of this jet pair is used as the final observable in this search. The signal events are expected to peak at the charged Higgs boson mass. We assume $\mathcal{B}\left(\mathrm{t} \rightarrow \mathrm{H}^{+} \mathrm{b}\right)+\mathcal{B}(\mathrm{t} \rightarrow \mathrm{Wb})=1.0$, which implies a lowering of the branching fraction of top quarks to $\mathrm{Wb}$ in presence of $\mathrm{H}^{+}$in top quark decays.

The main background for this search is $\mathrm{SM} t \overline{\mathrm{t}}$, including $\mathrm{t} \overline{\mathrm{t}}$ production in association with heavy-flavoured jets $(t \bar{t} b \bar{b}, t \bar{t} c \bar{c})$. Other considered backgrounds are single top production, multijet, $\mathrm{W} / \mathrm{Z}+$ jets and diboson production, and $\mathrm{t} \overline{\mathrm{t}}$ production in association with an $\mathrm{H} / \mathrm{Z} / \mathrm{W}$ boson.

\section{Event simulation and reconstruction with CMS detector}

Background samples of $\mathrm{t} \overline{\mathrm{t}}, \mathrm{t} \overline{\mathrm{t}}+\mathrm{W} / \mathrm{Z}$, and $\mathrm{W} / \mathrm{Z}+$ jets are simulated at leading order (LO) using the MADGRAPH 5.1 generator [45] with the CTEQ6L1 parton distribution function (PDF) set [46]. The top quark mass is set to $172.5 \mathrm{GeV}$ for simulating these samples. The predicted t $\overline{\mathrm{t}}$ production cross section is calculated with the Top ++2.0 program at the next-to-next-to-leading order (NNLO) in perturbative quantum chromodynamics 
(QCD), including soft-gluon resummation at the next-to-next-to-leading-log order (ref. [47] and references therein), to be $\sigma_{\mathrm{t} \overline{\mathrm{t}}}=252.9_{-8.6}^{+6.4}$ (scale) $\pm 11.7\left(\mathrm{PDF}+\alpha_{S}\right) \mathrm{pb}$, where "scale" and "PDF $+\alpha_{S}$ " refer to the uncertainties coming from the independent variation of the factorization and renormalization scales, and the variations in the PDF set and in the strong coupling constant $\alpha_{S}$, respectively, following the PDF4LHC prescription with the MSTW2008 68\% CL NNLO, CT10 NNLO and NNPDF2.3 5f FFN PDF sets (refs. [48, 49] and references therein, and refs. [50-52]).

The transverse momentum $p_{\mathrm{T}}$ distribution of top quarks in simulated t $\overline{\mathrm{t}}$ events is reweighted to match the $p_{\mathrm{T}}$ distribution observed in collision data [53]. The simulated $\mathrm{W} / \mathrm{Z}+$ jets samples are normalized to the NNLO cross section calculated with FEWZ 3.1 [54, $55]$, and $t \bar{t}+\mathrm{W} / \mathrm{Z}$ events are normalized to the next-to-leading order (NLO) cross section $[56,57]$. Single top quark events are generated with the POWHEG v1.0 generator [5861] and the CTEQ6M PDF set [46], and are normalized to the production cross section at NLO in QCD computed with HATHOR v2.1 [62, 63]. Diboson (WW/WZ/ZZ) and $t \bar{t} \mathrm{H}$ events are generated at LO using PYTHIA v6.4 [64] and normalized to the NLO cross section calculated using MCFM 6.6 [65] and the cross section given in ref. [66], respectively.

The charged Higgs boson signal events ( $\left.\mathrm{t} \overline{\mathrm{t}} \rightarrow \mathrm{bH}^{+} \overline{\mathrm{b}} \mathrm{W}^{-} \rightarrow \mathrm{b} \overline{\mathrm{b}} \mathrm{c} \overline{\mathrm{b}} \ell \nu\right)$ are simulated using the PYTHIA v6.4 and CTEQ6L1 PDF set for $M_{\mathrm{H}^{+}}=90,100,110,120,130,140$, and $150 \mathrm{GeV}$. These samples are normalized to the SM t $\overline{\mathrm{t}}$ cross section in lepton+jets channel. Consequently, in the assumption of $\mathcal{B}\left(\mathrm{H}^{+} \rightarrow \mathrm{cb}\right)=1.0$ and $\mathcal{B}\left(\mathrm{t} \rightarrow \mathrm{H}^{+} \mathrm{b}\right)+\mathcal{B}(\mathrm{t} \rightarrow \mathrm{Wb})=$ 1.0, a fit using templates of the SM tét and the $\mathrm{H}^{+}$signal determines the branching fraction of $\mathrm{t} \rightarrow \mathrm{H}^{+} \mathrm{b}$.

All generated samples are interfaced with PYTHIA v6.4 in order to simulate parton showering and hadronization, and then processed through the full simulation of the CMS detector based on GEant4 [67]. The underlying event tune Z2* [68, 69] is used. To ensure correct simulation of the number of additional interactions per bunch crossing (pileup), simulated events are mixed with multiple inelastic collision events and reweighted according to the distribution of the number of pileup interactions observed in data.

The central feature of the CMS apparatus is a superconducting solenoid of $6 \mathrm{~m}$ internal diameter, providing a magnetic field of $3.8 \mathrm{~T}$. Within the solenoid volume are a silicon pixel and strip tracker, a lead tungstate crystal electromagnetic calorimeter (ECAL), and a brass and scintillator hadron calorimeter (HCAL), each composed of a barrel and two endcap sections. Additional forward calorimetry complements the coverage provided by the barrel and endcap detectors. Muons are detected in gas-ionization chambers embedded in the steel flux-return yoke outside the solenoid. A more detailed description of the CMS detector, together with a definition of the coordinate system used and the relevant kinematic variables, can be found in ref. [70].

A particle-flow (PF) algorithm [71] aims to reconstruct and identify particle candidates with an optimized combination of information from various elements of the CMS detector. Muon momenta are obtained from the curvature of muon tracks. The energy of photons is obtained from the ECAL measurement, upon proper calibration of several instrumental effects as described in [72, 73]. The energy of electrons is determined from a combination of the electron momentum at the primary interaction vertex (PV) as determined by 
the tracker, the energy of the corresponding ECAL cluster, and the energy sum of all bremsstrahlung photons spatially compatible with originating from the electron track [74]. The $\mathrm{PV}$ is the reconstructed vertex with the largest value of $\sum p_{\mathrm{T}}^{2}$, the sum of squared transverse momenta of the charged particle tracks associated with the vertex. The energy of charged hadrons is determined from a combination of their momentum measured in the tracker and the matching ECAL and HCAL energy deposits. Finally, the neutral hadrons are identified as HCAL energy clusters not linked to any charged hadron trajectory, or as ECAL and HCAL energy excesses with respect to the expected charged hadron energy deposit or photon.

Jets are reconstructed from all the PF candidates clustered using the anti- $k_{\mathrm{T}}$ algorithm $[75,76]$ with a distance parameter of 0.5 . The jet momentum is determined as the vectorial sum of all particle momenta in the jet, and corrected for effects of pileup within the same or nearby bunch crossings. Jet energy scale corrections [77, 78] are used to account for the nonlinear energy response of the calorimeters and other instrumental effects. Additional selection criteria are applied to each event to remove spurious jet-like features originating from isolated noise patterns in certain HCAL regions. The missing transverse momentum vector $\vec{p}_{\mathrm{T}}^{\text {miss }}$ is defined as the projection onto the plane perpendicular to the beam axis of the negative vector sum of the momenta of all reconstructed PF objects in an event. Its magnitude is referred to as $p_{T}^{\text {miss }}$.

\section{Event selection and yields}

Candidate signal events are selected using triggers [79] that require a single isolated muon (electron) with $p_{\mathrm{T}}>24(27) \mathrm{GeV}$ and pseudorapidity $|\eta|<2.1$ (2.5). Further selection requirements are made offline. Events with exactly one muon (electron) with $p_{\mathrm{T}}>$ $26(30) \mathrm{GeV}$ and $|\eta|<2.1(2.5)$ are selected. Lepton identification selections, including requirements of a good track quality and close distance with respect to the PV, are imposed on each lepton candidate. Leptons must be isolated, satisfying relative isolation requirement $I_{\text {rel }}<0.12(0.1)$ for muons (electrons). The $I_{\text {rel }}$ is defined as the pileupcorrected scalar $p_{\mathrm{T}}$ sum around the lepton candidate's direction at the vertex divided by the lepton candidate $p_{\mathrm{T}}$. The $p_{\mathrm{T}}$ sum is calculated from momenta of the reconstructed charged hadrons originating from the PV, neutral hadrons, and photons within a cone of $\Delta R=\sqrt{(\Delta \eta)^{2}+(\Delta \phi)^{2}}<0.4$ (0.3) for muons (electrons), where $\phi$ is the azimuthal opening angle (in radians). Events with any additional muons (electrons) satisfying $p_{\mathrm{T}}>10(20)$, $|\eta|<2.5$, and $I_{\text {rel }}<0.3$, are discarded.

The $p_{\mathrm{T}}^{\text {miss }}$ is required to be larger than $20 \mathrm{GeV}$, and at least four jets are required to have $p_{\mathrm{T}}>30 \mathrm{GeV}$ within the tracker coverage of $|\eta|<2.4$. To identify jets originating from b quarks, the combined secondary vertex tagging algorithm [80] is used. Selected jets are considered b-tagged if they satisfy the medium working point requirements of this algorithm. This results in an efficiency of approximately $70 \%$ for tagging a b quark jet, and a mistag rate of $1 \%$ for light quark and gluon jets. The probability of a $\mathrm{c}$ jet to be tagged as a b jet is about $20 \%$. Events with two or more b-tagged jets are selected. 
The events selected using the above criteria are dominated by SM t $\overline{\mathrm{t}}$ events $(\approx 92 \%)$ based on the background simulation samples. The observed event yields in events with two b-tagged jets are well described by the simulation, however, the events containing three or more b-tagged jets are more difficult to model. In order to estimate the t $\overline{\mathrm{t}}$ component in the three or more b-tagged jet event sample, we rely on the measurement of the t $\bar{t} b \bar{b}$ cross section in ref. [81]. In this reference, the t $\bar{t} b \bar{b}$ cross section is measured to be $0.36 \pm 0.08$ (stat) \pm 0.1 (syst) pb. Comparing with the theoretical expectation of $0.23 \pm 0.05 \mathrm{pb}$, we obtain a ratio between the measured and the expected tt̄b $\bar{b}$ production cross section of $1.56 \pm 0.66$. As the study used dilepton $t \bar{t}$ events of same generator with current $t \bar{t}$ simulation sample, in which both top quarks decay to $\mathrm{Wb}$ with $\mathrm{W} \rightarrow \ell \nu$, the $\mathrm{H}^{+}(\rightarrow c \bar{b})$ contribution to this extra

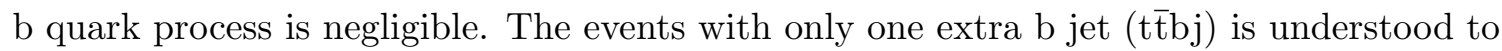
come from the $t \bar{t} b \bar{b}$ process with one $b$ jet missed. Consequently, the $t \bar{t} b \bar{b}$ component in the simulated $t \bar{t}$ sample is estimated by requiring at least one additional jet originated from an

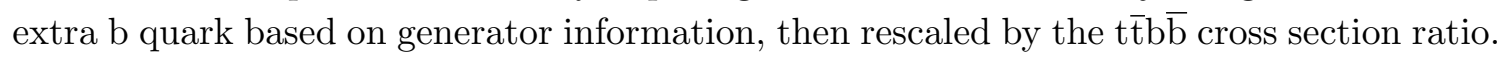

The multijet background is estimated following the method used in ref. [38]. The shapes of the multijet background distributions are obtained from a nonisolated control region defined by $0.15<I_{\text {rel }}<0.3$ and $p_{\mathrm{T}}^{\text {miss }}>20 \mathrm{GeV}$, after subtraction of the estimated SM backgrounds. In a QCD enhanced control region $\left(p_{\mathrm{T}}^{\text {miss }}<20 \mathrm{GeV}\right)$, a multiplicative scale factor used for the multijet background normalization is obtained from the nonisolated control region extrapolated to the isolated region. The shape uncertainty is estimated from the multijet background samples obtained using the same method but with shifted nonisolated control regions, $0.2<I_{\text {rel }}<0.3$ (smaller statistics) and reversing $I_{\text {rel }}$ selection, $0.12(\mu) / 0.1(\mathrm{e})<I_{\text {rel }}<0.3$ (larger statistics). The normalization uncertainty is estimated by an average difference in the multijet background yields obtained from the shifted nonisolated control regions compared to the nominal multijet background, and its impact on

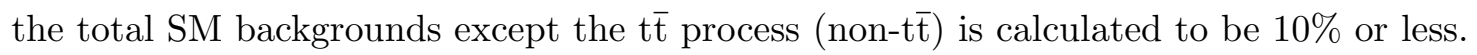

Event yields satisfying the selection criteria in the absence of a signal are summarized in table 1 . The $t \bar{t}$ event yields are estimated after rescaling the $t \bar{t} b \bar{b}$ component. The number of b-tagged jets ( $\mathrm{b}$ tags) indicated in table 1 is the number of $\mathrm{b}$ tags among the

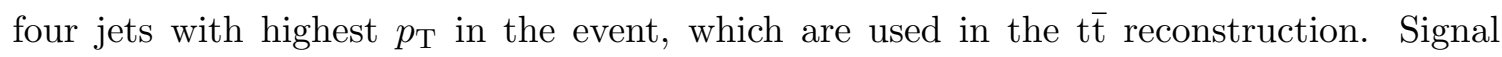
efficiency satisfying the selection criteria is $4-6 \%$ depending on $M_{\mathrm{H}^{+}}$.

\section{Reconstruction of $t \bar{t}$ events}

Top quark and $\mathrm{W}$ boson masses are reconstructed relying on the knowledge of the momenta of their decay products. However, the reconstructed mass is different from the true mass because the measured jet energy is corrected to the energy of a particle-level jet, not to the energy of the initial parton. A correction is derived from the energy shift between a particle-level jet and the matched hard scattering parton within $\Delta R=0.3$, depending on

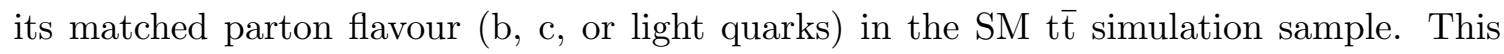
correction is called the top quark specific (TS) correction and is applied as a function of the $p_{\mathrm{T}}$ and $\eta$ of the jet. The application of TS correction in the $t \overline{\mathrm{t}}$ reconstruction have been used in several analyses [35, 37, 82]. Using this correction increases the accuracy of 


\begin{tabular}{|c|c|c|c|c|}
\hline & \multicolumn{2}{|c|}{$\mu+$ jets } & \multicolumn{2}{|c|}{ e+jets } \\
\hline & $2 \mathrm{~b}$ tags & $\geq 3 \mathrm{~b}$ tags & $2 \mathrm{~b}$ tags & $\geq 3 \mathrm{~b}$ tags \\
\hline $\mathrm{t} \overline{\mathrm{t}}$ & $52821 \pm 67 \pm 5463$ & $5060 \pm 21 \pm 586$ & $44484 \pm 60 \pm 4682$ & $4269 \pm 19 \pm 468$ \\
\hline Single top & $2212 \pm 30 \pm 178$ & $169 \pm 8 \pm 16$ & $1882 \pm 28 \pm 161$ & $147 \pm 8 \pm 13$ \\
\hline $\mathrm{t} \overline{\mathrm{t}}+\mathrm{W} / \mathrm{Z} / \mathrm{H}$ & $195 \pm 2 \pm 8$ & $41 \pm 1 \pm 3$ & $169 \pm 2 \quad \pm 7$ & $35 \pm 1 \pm 2$ \\
\hline $\mathrm{W} / \mathrm{Z}+$ jets & $1305 \pm 127 \pm 157$ & $13 \pm 7 \pm 13$ & $1098 \pm 114 \pm 165$ & $32 \pm 19 \pm 14$ \\
\hline $\mathrm{WW} / \mathrm{WZ} / \mathrm{ZZ}$ & $62 \pm 2 \quad \pm 7$ & $5 \pm 1 \pm 1$ & $56 \pm 2 \pm 6$ & $4 \pm 1 \pm 1$ \\
\hline Multijet & $497 \pm 15 \pm 15$ & $190 \pm 19 \pm 23$ & $996 \pm 31 \pm 58$ & $178 \pm 17 \pm 20$ \\
\hline Expected & $57093 \pm 5470$ (stat+syst) & $5477 \pm 588$ (stat+syst) & $48683 \pm 4688$ (stat+syst) & $4665 \pm 470$ (stat+syst) \\
\hline Observed & 57593 & 5754 & 50542 & 4848 \\
\hline
\end{tabular}

Table 1. Observed event yields and estimated backgrounds for the $\mu+$ jets and e+jets channels satisfying the event selection criteria. The number of b-tagged jets is the number of b tags among the four jets with highest $p_{\mathrm{T}}$ in the event. The first and second uncertainty shown corresponds to the statistical and systematic components, respectively.

the mass reconstruction for top quarks and $\mathrm{H}^{+} / \mathrm{W}$ boson decaying to dijet, resulting in a 7-9\% improvement in resolution.

The instrumental mass resolution is further improved using a kinematic fit. The fit is used to fully reconstruct the $t \overline{\mathrm{t}}$ system by assigning selected jets to the hadronic $\mathrm{W} / \mathrm{H}^{+}$ decays or $b$ quarks in $t \bar{t}$ decays. The function that is minimized in the fit is as follows:

$$
\begin{aligned}
\chi^{2}=\sum_{p_{z}^{\nu} \text { solutions }}\left(\sum_{i=\ell, 4 \text { jets }} \frac{\left(p_{\mathrm{T}}^{i, \mathrm{fit}}-p_{\mathrm{T}}^{i, \text { meas }}\right)^{2}}{\sigma_{i}{ }^{2}}+\sum_{j=x, y} \frac{\left(p_{j}^{\mathrm{UE}, \mathrm{fit}}-p_{j}^{\mathrm{UE}, \mathrm{meas}}\right)^{2}}{\sigma_{\mathrm{UE}}{ }^{2}}\right. \\
\left.+\frac{\left(M_{\ell \nu}-M_{\mathrm{W}}\right)^{2}}{\Gamma_{\mathrm{W}}{ }^{2}}+\sum_{k=\mathrm{t}^{\text {had }}, \mathrm{t}_{\text {lep }}} \frac{\left(M_{k}-M_{\mathrm{t}}\right)^{2}}{\Gamma_{\mathrm{t}}{ }^{2}}\right) .
\end{aligned}
$$

In the first two terms, the momentum with superscript "fit" is the variable to be determined by the fit, and the measured TS-corrected input $p_{\mathrm{T}}$ is denoted with the superscript "meas". The first term fits the transverse momentum of the lepton and leading four jets and the second term fits an unclustered energy (UE) in the transverse directions $x$ and $y$. The unclustered transverse energy vector is obtained from all the observables in the transverse plane by the relation:

$$
p_{x, y}^{\mathrm{UE}}=-\sum_{i=\ell, 4 \text { jets }} p_{x, y}^{i}-\sum_{j=\text { extra jets, } p_{\mathrm{T}}>10 \mathrm{GeV},|\eta|<2.5} p_{x, y}^{j}-p_{x, y}^{\text {miss }},
$$

where the $p_{x}^{\text {miss }}$ and $p_{y}^{\text {miss }}$ are the $x$ and $y$ components of $\vec{p}_{\mathrm{T}}^{\text {miss }}$. Variation of the lepton, jet, and UE is allowed within the measurement uncertainties, $\sigma_{i}$ and $\sigma_{\mathrm{UE}}$, depending on their $p_{\mathrm{T}}$. The longitudinal momentum $\left(p_{z}^{\nu}\right)$ of the neutrino is calculated by the leptonic $(\ell \nu) \mathrm{W}$ boson mass constraint $\left(\left[\mathbf{p}^{\ell}+\mathbf{p}^{\nu}\right]^{2}=M_{\mathrm{W}}^{2}\right)$ and only real $p_{z}^{\nu}$ is taken into account in the fit. During the iterations for minimizing the $\chi^{2}$, this $p_{z}^{\nu}$ varies to keep the $\mathrm{W}$ boson mass constrained. The neutrino momentum vector $\left(p_{x}^{\nu, \mathrm{fit}}, p_{y}^{\nu, \mathrm{fit}}, p_{z}^{\nu, \mathrm{fit}}\right)$ is reconstructed from all the fitted momenta and eq. 4.2: $p_{x, y}^{\nu, \text { fit }}=p_{x, y}^{\text {miss,fit }}$. The last term constrains the hadronic and leptonic top quark candidates to have the true mass of $172.5 \mathrm{GeV}$. The widths of the $\mathrm{W}$ 
boson $\left(\Gamma_{\mathrm{W}}\right)$ and top quark $\left(\Gamma_{\mathrm{t}}\right)$ in ref. [19] are used for the resolution in the fit. The $\chi^{2}$ minimization is performed for each possible combination of the four leading jets to quarks in the $t \bar{t}$ system, where the b-tagged jets are only assigned to the $\mathrm{b}$ quark daughters. In order to suppress combinatorial backgrounds and the irreducible contaminations from initial-

and final-state radiation jets, two requirements are imposed: $\left|p_{\mathrm{T}}^{\text {jet, meas }}-p_{\mathrm{T}}^{\text {jet, fit }}\right|<20 \mathrm{GeV}$ for the jets used in the fit and $M_{k}<200 \mathrm{GeV}$, in which $M_{k}$ is reconstructed using input jets before the $\chi^{2}$ fit, for the hadronically decaying top quark. In the jet-quark assignment that minimizes the $\chi^{2}$, the two jets not assigned to either $\mathrm{b}$ quarks originating directly from top quark decays form a $\mathrm{H}^{+} \rightarrow c \bar{b}$ candidate.

The reconstructed events are further categorized according to the lepton flavour ( $\mu$ or e) and the number of b-tagged jets ( 2 or $\geq 3$ ). Events containing two b-tagged jets are

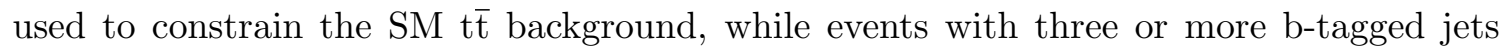
are used to search directly the presence of $\mathrm{H}^{+} \rightarrow c \bar{b}$ decays. In events with two $\mathrm{b}$ tags, the fit has only two possible combinations of the jet assignment. However, in events with three or more b tags, one b-tagged jet is assigned to a leptonically decaying top quark, and two other b-tagged jets are assigned to the hadronically decaying top quark resulting in additional ambiguity. According to simulation, the ambiguity is efficiently resolved by the fit procedure only for $\mathrm{H}^{+}$masses below $120 \mathrm{GeV}$. At higher masses $(130-150 \mathrm{GeV})$, the ambiguity is resolved by assigning the $\mathrm{b}$ jet with the lower $p_{\mathrm{T}}$ to the $\mathrm{b}$ quark that originates from the $\mathrm{t} \rightarrow \mathrm{H}^{+} \mathrm{b}$ decay.

\section{$5 \quad$ Systematic uncertainties}

Systematic uncertainties can affect the overall signal and background events, as well as cause distortions in the shape of the dijet mass distribution. Since the $\mathrm{H}^{+}$originates from

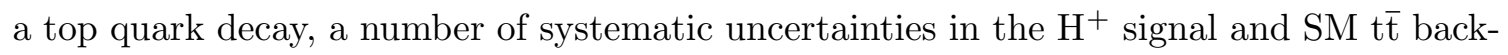
ground are correlated. The systematic uncertainties are estimated based on the samples and methods used in ref. [83]. A summary of the systematic uncertainties is given in table 2 .

Sources of systematic uncertainties are grouped into several categories: jet corrections, $\mathrm{b}$ tagging effects, $\mathrm{t} \overline{\mathrm{t}}$ modeling, and normalizations. Uncertainties due to jet energy corrections, flavour-dependent uncertainties, and uncertainties due to jet energy resolution corrections are estimated by varying the correction factors by \pm 1 standard deviation (s.d.). The efficiency difference from data to the simulation (scale factor) in heavy quark tagging (b/c jets) and mistagging for light-flavoured jets is also varied by \pm 1 s.d. separately and the corresponding changes are estimated. Similarly, the following quantities are also varied by \pm 1 s.d.: normalization of the $t \bar{t}$ cross section in the simulation, integrated luminosity [84] of the data sample, and lepton scale factors including the single-lepton trigger, identification, and relative isolation. The uncertainty due to pileup is estimated by varying the total inelastic cross section used in the simulation by $\pm 5 \%$ [81].

To account for the uncertainties in the modeling of SM t $\bar{t}$ events, we consider the un-

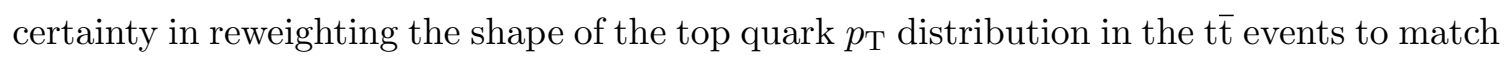
the simulation to data, NLO production versus LO production with 0-3 partons (POWHEG versus MADGRAPH), matching thresholds used for interfacing the matrix-elements calcula- 


\begin{tabular}{|c|c|c|c|c|c|c|}
\hline \multirow{2}{*}{\begin{tabular}{|l} 
Source of uncertainty \\
Jet energy scale (JES)*
\end{tabular}} & \multicolumn{2}{|c|}{$\begin{array}{l}\text { Signal }\left(M_{\mathrm{H}^{+}}=120 \mathrm{GeV}\right)(\%) \\
2 \mathrm{~b} \text { tags } \geq 3 \mathrm{~b} \text { tags }\end{array}$} & \multicolumn{2}{|c|}{$\begin{array}{c}\mathrm{t} \overline{\mathrm{t}}(\%) \\
2 \mathrm{~b} \text { tags } \geq 3 \mathrm{~b} \text { tags }\end{array}$} & \multicolumn{2}{|c|}{ 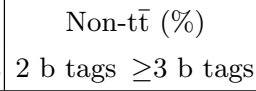 } \\
\hline & $4.6-5.3$ & $5.0-5.8$ & $3.1-3.3$ & 3.1 & $10.2-14.5$ & $1.9-3.4$ \\
\hline Flavour-dependent JES (b quark)* & 0.4 & 0.5 & 0.1 & 0.1 & 0.2 & $0.5-3.4$ \\
\hline Flavour-dependent JES (udsc quark or gluon)* & 1.0 & 0.4 & 0.9 & 0.8 & $2.8-4.6$ & $2.7-9.0$ \\
\hline Jet energy resolution* & 0.2 & 0.8 & 0.3 & 0.3 & $1.0-1.3$ & $1.3-4.9$ \\
\hline b tagging scale factor for b/c-quark jets* & 1.2 & 5.7 & 3.6 & 5.7 & $0.6-0.8$ & $2.0-3.8$ \\
\hline Mistag scale factor for light quark jets* & 0.2 & 0.3 & 0.2 & 2.7 & $0.9-1.5$ & $0.9-2.0$ \\
\hline $\mathrm{t} \overline{\mathrm{t}} p_{\mathrm{T}}$ reweighting* & 0.2 & 1.0 & $1.4-1.7$ & $1.6-1.9$ & - & - \\
\hline NLO-vs.-LO shape* & $7.5-8.4$ & $7.2-7.7$ & $7.0-8.2$ & $6.8-7.6$ & - & - \\
\hline ME-PS matching* & 0.8 & 0.9 & 1.1 & $1.8-2.4$ & - & - \\
\hline Renormalization and factorization scales* & 0.3 & $1.3-1.8$ & $0.8-1.8$ & $1.3-1.6$ & - & - \\
\hline Top quark mass* & $1.1-1.4$ & $1.1-1.5$ & $0.4-1.2$ & 0.9 & - & - \\
\hline $\mathrm{t} \overline{\mathrm{tb}} \overline{\mathrm{b}}$ production rescaling* & - & - & $3.7-3.9$ & $10.2-10.9$ & - & - \\
\hline PYTHIA-MADGRAPH $p_{\mathrm{T}}(\mathrm{t} \overline{\mathrm{t}})$ difference* & 0.1 & 0.1 & - & - & - & - \\
\hline $\mathrm{t} \overline{\mathrm{t}}$ cross section & 6.5 & 6.5 & 6.5 & 6.5 & - & - \\
\hline Integrated luminosity & & & 2.6 & & & \\
\hline Muon scale factor $(\mu+$ jets $)$ & & & 3.0 & & & \\
\hline Electron scale factor $(\mathrm{e}+$ jets $)$ & & & 3.0 & & & \\
\hline Pileup reweighting & & & $0.1-1.3$ & & & \\
\hline Multijet background prediction from data* & & - & & & $0.3-2.3$ & $5.2-10.7$ \\
\hline
\end{tabular}

Table 2. Summary of the relative systematic uncertainties in the event yields for the $\mathrm{H}^{+}$signal

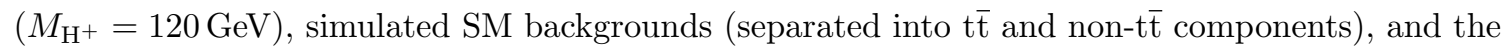
data-driven multijet events. The uncertainties apply to both $\mu+$ jets and e+jets events, and in the case where the uncertainties in the two channels differ, a range is given. Uncertainties on the shape of templates are marked with an asterisk.

tions of the MADGRAPH generator to the PYTHIA parton showers (ME-PS), renormalization and factorization scales, and the uncertainty in the top quark mass of $172.5 \pm 1.0 \mathrm{GeV}$. The

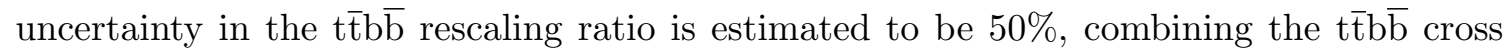
section uncertainties $(42 \%)$ and a few percent of the inefficiency of counting b jets in generator level. The $t \bar{t} b \bar{b}$ rescaling uncertainties listed in table 2 are the impact of rescaling on the selected $t \bar{t}$ events.

The systematic uncertainty in the SM t $\bar{t}$ modeling is estimated using simulation samples in which the corresponding systematic sources are varied. In order to estimate the t $\overline{\mathrm{t}}$ modeling uncertainties in the simulated $\mathrm{H}^{+}$signal events, the $p_{\mathrm{T}}$ distribution of the top quarks from SM t $\overline{\mathrm{t}}$ events is used. The ratio of the $p_{\mathrm{T}}$ distribution with each parameter shifted to the nominal value is calculated, then is used to reweight the top quark $p_{\mathrm{T}}$ distributions in the $\mathrm{H}^{+}$signal simulation to mimic the systematic sample. By using this method the modeling uncertainties for $\mathrm{H}^{+}$signal events are estimated as listed in table 2 .

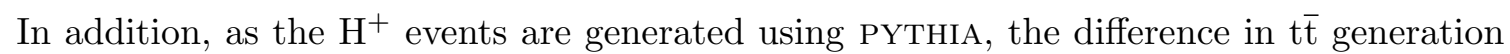
estimated by the top quark $p_{\mathrm{T}}$ distributions of PYTHIA and MADGRAPH, is then used as an additional systematic uncertainty. 

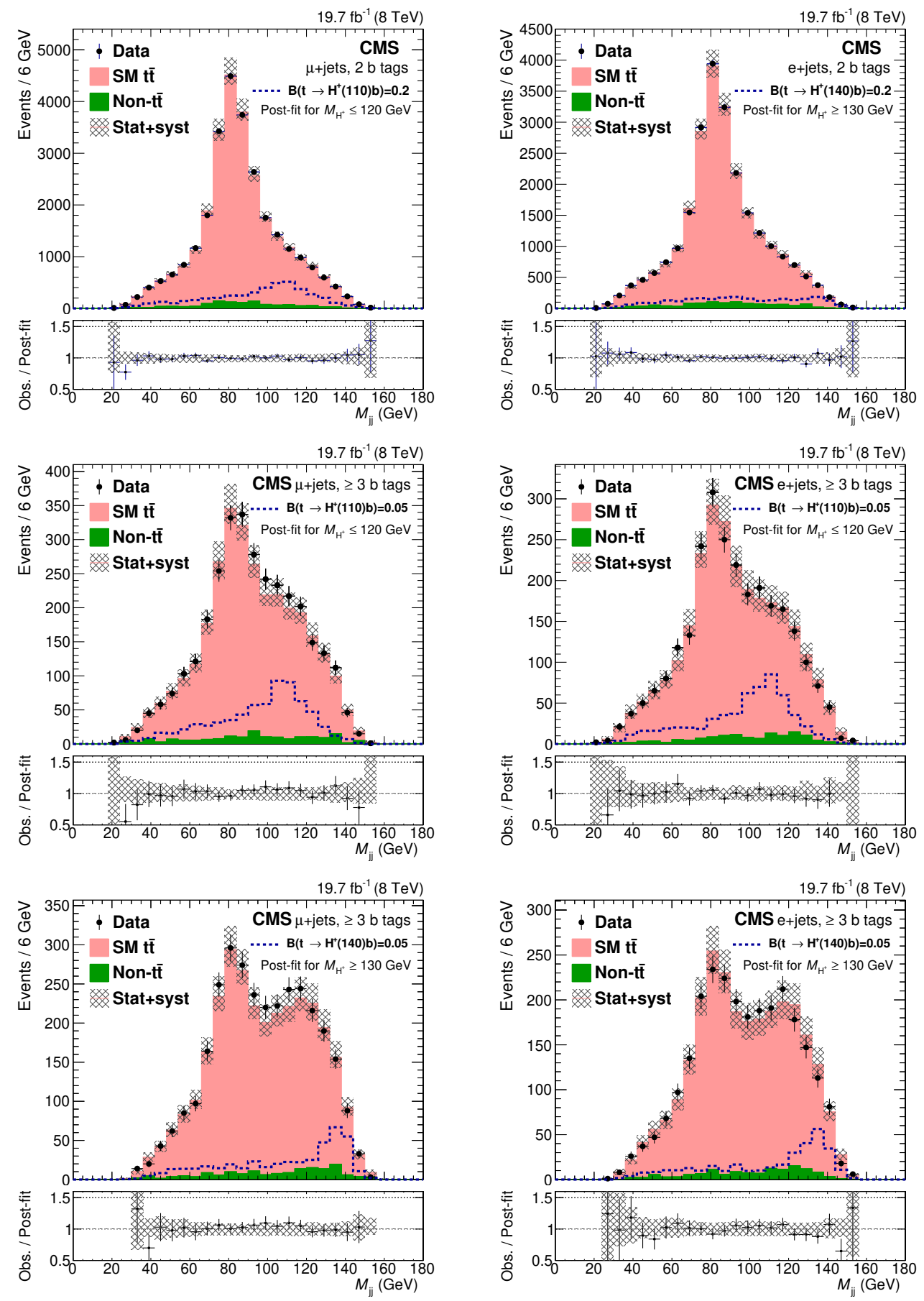

Figure 2. Post-fit with a null- $\mathrm{H}^{+}$hypothesis on the expected dijet mass distributions from SM backgrounds (cumulative filled histograms) and their ratio of observed to predicted yields for the $\mu+$ jets (left column) and e+jets (right column) channels. In the first row, events are shown for two $\mathrm{b}$ tags together with the fit procedure for a $\mathrm{H}^{+} \operatorname{signal}\left(M_{\mathrm{H}^{+}}=110 \mathrm{GeV}\right.$ in left and $140 \mathrm{GeV}$ in right). The second (third) row shows the results for events with at least three $\mathrm{b}$ tags in the fit procedure for the $\mathrm{H}^{+}$search with $M_{\mathrm{H}^{+}}=90-120(130-150) \mathrm{GeV}$. The dijet distributions are compared with the $\mathrm{H}^{+}$signal shape (dashed line) for $M_{\mathrm{H}^{+}}=110$ and $140 \mathrm{GeV}$. 


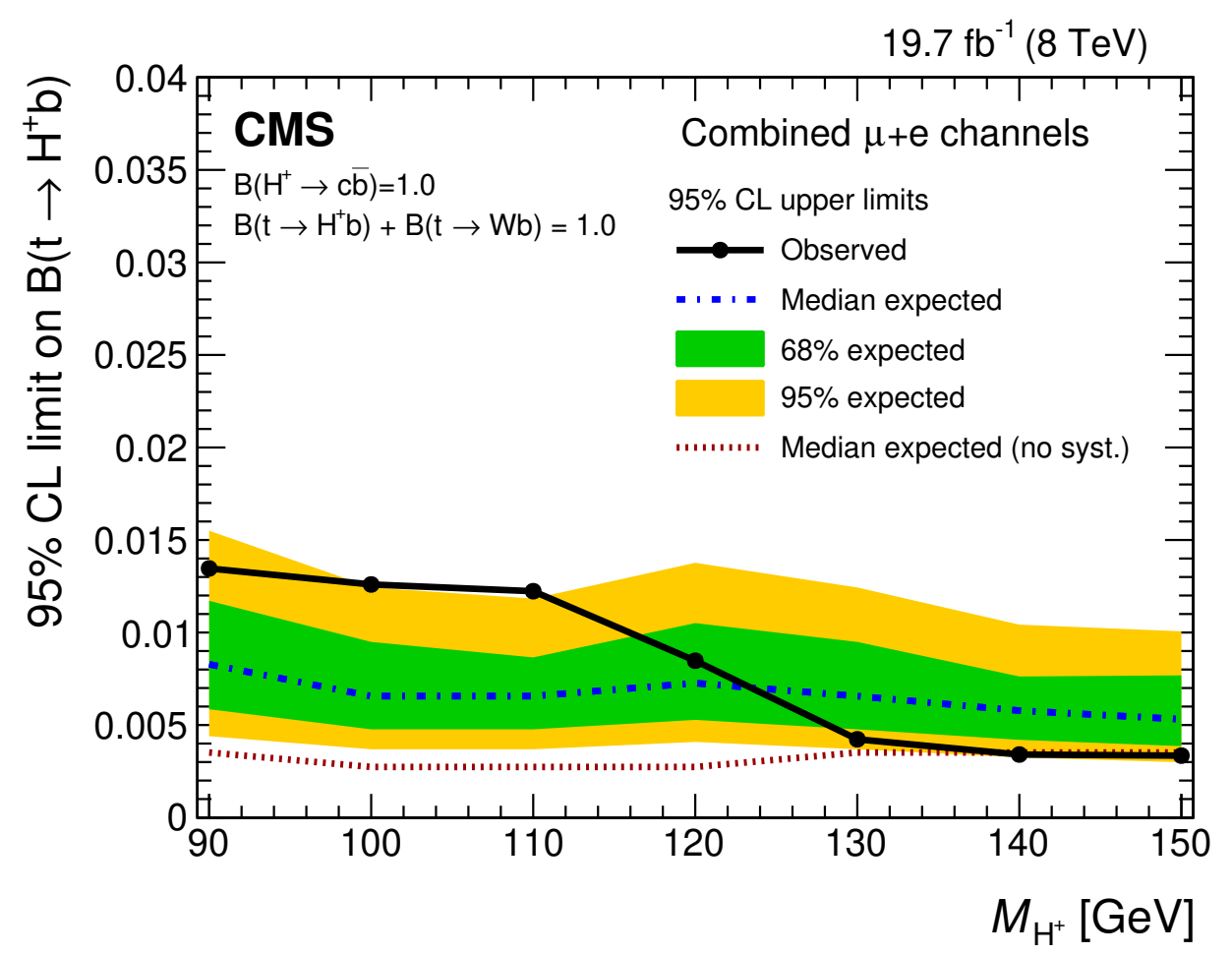

Figure 3. Upper limits at the $95 \%$ confidence level $(\mathrm{CL})$ on the branching fraction $\mathcal{B}\left(\mathrm{t} \rightarrow \mathrm{H}^{+} \mathrm{b}\right)$, assuming $\mathcal{B}\left(\mathrm{H}^{+} \rightarrow \mathrm{cb}\right)=1.0$ and $\mathcal{B}\left(\mathrm{t} \rightarrow \mathrm{H}^{+} \mathrm{b}\right)+\mathcal{B}(\mathrm{t} \rightarrow \mathrm{Wb})=1.0$, for the combined $\mu+$ jets and e+jets channels. The black solid line shows the observed limit. The mean expected limit is shown as a blue dashed line and the green/yellow bands indicate the $68 / 95 \%$ confidence intervals for the expected limits. The red dotted line shows the mean expected limit in the absence of systematic uncertainties.

\section{Results}

Figure 2 shows the dijet mass distributions together with the expected SM processes and $\mathrm{H}^{+}$ signal after the kinematic fit procedures in $\mu+$ jets and e+jets events with two $\mathrm{b}$ tags and at least three b tags, which are used for the $\mathrm{H}^{+}$search with $M_{\mathrm{H}^{+}}$of $90-120$ and $130-150 \mathrm{GeV}$. A binned maximum likelihood fit is performed simultaneously to all the observed dijet mass distributions, using the signal and background templates extracted from the simulation or from the data. The background templates are composed of the dominant SM t $\bar{t}$ and non-t $\overline{\mathrm{t}}$ contributions. For the $M_{\mathrm{H}^{+}}$values of 120 and $130 \mathrm{GeV}$, where the kinematic fit procedure changes as described in section 4 , the limits are derived also with the alternate procedure, giving consistent results. No significant excess is seen above the expected SM background. The upper limits at $95 \% \mathrm{CL}$ on the branching fraction $\mathcal{B}\left(\mathrm{t} \rightarrow \mathrm{H}^{+} \mathrm{b}\right)$ are calculated using the statistical tools in RooStAT [85] and the $\mathrm{CL}_{\mathrm{s}}$ criterion [86, 87] with a profile likelihood ratio as a test statistic [88] and using an asymptotic formulae [89]. The expected branching fraction limit is calculated using an Asimov dataset with a null hypothesis. Systematic uncertainties are treated as nuisance parameters and profiled in the fit following a log- 
normal distribution for the normalization uncertainties and using distorted templates for shape systematic uncertainties. With the assumptions of $\mathcal{B}\left(\mathrm{H}^{+} \rightarrow \mathrm{c} \overline{\mathrm{b}}\right)=1.0$ and $\mathcal{B}(\mathrm{t} \rightarrow$ $\left.\mathrm{H}^{+} \mathrm{b}\right)+\mathcal{B}(\mathrm{t} \rightarrow \mathrm{Wb})=1.0$, the expected and observed limits as a function of $M_{\mathrm{H}^{+}}$are shown in figure 3 . The expected limits without systematic uncertainties are also shown to illustrate that the analysis sensitivity is largely limited by the present level of our knowledge of the systematic uncertainties. The biggest impact on the expected limit comes from the $\mathrm{t} \overline{\mathrm{t}} \mathrm{b} \overline{\mathrm{b}}$ production rescaling uncertainty.

\section{Summary}

A search for charged Higgs boson decaying to a charm and a bottom quark $\left(\mathrm{H}^{+} \rightarrow \mathrm{c} \overline{\mathrm{b}}\right)$ is performed for the first time. The search uses t $\bar{t}$ events with a final state containing at least four jets, a charged lepton (muon or electron), and missing transverse momentum. The search is based on the analysis of proton-proton collision data recorded at $\sqrt{s}=8 \mathrm{TeV}$, corresponding to an integrated luminosity of $19.7 \mathrm{fb}^{-1}$. A kinematic fit is performed to identify the pair of jets least likely to be the $b$ quarks originating from direct top quark decays and the invariant mass of this pair is used as the final observable in the search. No evidence for the presence of a charged Higgs boson is observed and upper limits at $95 \%$ confidence level of $0.8-0.5 \%$ are set on the branching fraction $\mathcal{B}\left(\mathrm{t} \rightarrow \mathrm{H}^{+} \mathrm{b}\right)$, assuming $\mathcal{B}\left(\mathrm{H}^{+} \rightarrow \mathrm{c} \overline{\mathrm{b}}\right)=1.0$ and $\mathcal{B}\left(\mathrm{t} \rightarrow \mathrm{H}^{+} \mathrm{b}\right)+\mathcal{B}(\mathrm{t} \rightarrow \mathrm{Wb})=1.0$, for the charged Higgs boson mass range $90-150 \mathrm{GeV}$.

\section{Acknowledgments}

We congratulate our colleagues in the CERN accelerator departments for the excellent performance of the LHC and thank the technical and administrative staffs at CERN and at other CMS institutes for their contributions to the success of the CMS effort. In addition, we gratefully acknowledge the computing centres and personnel of the Worldwide LHC Computing Grid for delivering so effectively the computing infrastructure essential to our analyses. Finally, we acknowledge the enduring support for the construction and operation of the LHC and the CMS detector provided by the following funding agencies: the Austrian Federal Ministry of Science, Research and Economy and the Austrian Science Fund; the Belgian Fonds de la Recherche Scientifique, and Fonds voor Wetenschappelijk Onderzoek; the Brazilian Funding Agencies (CNPq, CAPES, FAPERJ, and FAPESP); the Bulgarian Ministry of Education and Science; CERN; the Chinese Academy of Sciences, Ministry of Science and Technology, and National Natural Science Foundation of China; the Colombian Funding Agency (COLCIENCIAS); the Croatian Ministry of Science, Education and Sport, and the Croatian Science Foundation; the Research Promotion Foundation, Cyprus; the Secretariat for Higher Education, Science, Technology and Innovation, Ecuador; the Ministry of Education and Research, Estonian Research Council via IUT23-4 and IUT236 and European Regional Development Fund, Estonia; the Academy of Finland, Finnish Ministry of Education and Culture, and Helsinki Institute of Physics; the Institut National de Physique Nucléaire et de Physique des Particules / CNRS, and Commissariat à l'Énergie Atomique et aux Énergies Alternatives / CEA, France; the Bundesministerium für 
Bildung und Forschung, Deutsche Forschungsgemeinschaft, and Helmholtz-Gemeinschaft Deutscher Forschungszentren, Germany; the General Secretariat for Research and Technology, Greece; the National Research, Development and Innovation Fund, Hungary; the Department of Atomic Energy and the Department of Science and Technology, India; the Institute for Studies in Theoretical Physics and Mathematics, Iran; the Science Foundation, Ireland; the Istituto Nazionale di Fisica Nucleare, Italy; the Ministry of Science, ICT and Future Planning, and National Research Foundation (NRF), Republic of Korea; the Lithuanian Academy of Sciences; the Ministry of Education, and University of Malaya (Malaysia); the Mexican Funding Agencies (BUAP, CINVESTAV, CONACYT, LNS, SEP, and UASLP-FAI); the Ministry of Business, Innovation and Employment, New Zealand; the Pakistan Atomic Energy Commission; the Ministry of Science and Higher Education and the National Science Centre, Poland; the Fundação para a Ciência e a Tecnologia, Portugal; JINR, Dubna; the Ministry of Education and Science of the Russian Federation, the Federal Agency of Atomic Energy of the Russian Federation, Russian Academy of Sciences, the Russian Foundation for Basic Research and the Russian Competitiveness Program of NRNU "MEPhI"; the Ministry of Education, Science and Technological Development of Serbia; the Secretaría de Estado de Investigación, Desarrollo e Innovación, Programa Consolider-Ingenio 2010, Plan Estatal de Investigación Científica y Técnica y de Innovación 2013-2016, Plan de Ciencia, Tecnología e Innovación 2013-2017 del Principado de Asturias and Fondo Europeo de Desarrollo Regional, Spain; the Swiss Funding Agencies (ETH Board, ETH Zurich, PSI, SNF, UniZH, Canton Zurich, and SER); the Ministry of Science and Technology, Taipei; the Thailand Center of Excellence in Physics, the Institute for the Promotion of Teaching Science and Technology of Thailand, Special Task Force for Activating Research and the National Science and Technology Development Agency of Thailand; the Scientific and Technical Research Council of Turkey, and Turkish Atomic Energy Authority; the National Academy of Sciences of Ukraine, and State Fund for Fundamental Researches, Ukraine; the Science and Technology Facilities Council, U.K.; the U.S. Department of Energy, and the U.S. National Science Foundation.

Individuals have received support from the Marie-Curie programme and the European Research Council and Horizon 2020 Grant, contract No. 675440 (European Union); the Leventis Foundation; the A. P. Sloan Foundation; the Alexander von Humboldt Foundation; the Belgian Federal Science Policy Office; the Fonds pour la Formation à la Recherche dans l'Industrie et dans l'Agriculture (FRIA-Belgium); the Agentschap voor Innovatie door Wetenschap en Technologie (IWT-Belgium); the F.R.S.-FNRS and FWO (Belgium) under the "Excellence of Science - EOS" — be.h project n. 30820817; the Ministry of Education, Youth and Sports (MEYS) of the Czech Republic; the Lendület ("Momentum") Programme and the János Bolyai Research Scholarship of the Hungarian Academy of Sciences, the New National Excellence Program ÚNKP, the NKFIA research grants 123842, 123959, 124845, 124850 and 125105 (Hungary); the Council of Scientific and Industrial Research, India; the HOMING PLUS programme of the Foundation for Polish Science, cofinanced from European Union, Regional Development Fund, the Mobility Plus programme of the Ministry of Science and Higher Education, the National Science Center (Poland), contracts Harmonia 2014/14/M/ST2/00428, Opus 2014/13/B/ST2/02543, 2014/15/B/ST2/03998, 
and 2015/19/B/ST2/02861, Sonata-bis 2012/07/E/ST2/01406; the National Priorities Research Program by Qatar National Research Fund; the Programa de Excelencia María de Maeztu and the Programa Severo Ochoa del Principado de Asturias; the Thalis and Aristeia programmes cofinanced by EU-ESF and the Greek NSRF; the Rachadapisek Sompot Fund for Postdoctoral Fellowship, Chulalongkorn University and the Chulalongkorn Academic into Its 2nd Century Project Advancement Project (Thailand); the Welch Foundation, contract C-1845; and the Weston Havens Foundation (U.S.A.).

Open Access. This article is distributed under the terms of the Creative Commons Attribution License (CC-BY 4.0), which permits any use, distribution and reproduction in any medium, provided the original author(s) and source are credited.

\section{References}

[1] ATLAS collaboration, Observation of a new particle in the search for the Standard Model Higgs boson with the ATLAS detector at the LHC, Phys. Lett. B 716 (2012) 1 [arXiv: 1207.7214] [INSPIRE].

[2] CMS collaboration, Observation of a new boson at a mass of $125 \mathrm{GeV}$ with the CMS experiment at the LHC, Phys. Lett. B 716 (2012) 30 [arXiv:1207.7235] [INSPIRE].

[3] CMS collaboration, Observation of a new boson with mass near $125 \mathrm{GeV}$ in pp collisions at $\sqrt{s}=7$ and $8 \mathrm{TeV}$, JHEP 06 (2013) 081 [arXiv: 1303.4571] [INSPIRE].

[4] CMS collaboration, Precise determination of the mass of the Higgs boson and tests of compatibility of its couplings with the Standard Model predictions using proton collisions at 7 and $8 \mathrm{TeV}$, Eur. Phys. J. C 75 (2015) 212 [arXiv: 1412.8662] [INSPIRE].

[5] ATLAS and CMS collaborations, Combined measurement of the Higgs boson mass in pp collisions at $\sqrt{s}=7$ and 8 TeV with the ATLAS and CMS experiments, Phys. Rev. Lett. 114 (2015) 191803 [arXiv: 1503.07589] [INSPIRE].

[6] ATLAS collaboration, Measurements of the Higgs boson production and decay rates and coupling strengths using pp collision data at $\sqrt{s}=7$ and $8 \mathrm{TeV}$ in the ATLAS experiment, Eur. Phys. J. C 76 (2016) 6 [arXiv:1507.04548] [inSPIRE].

[7] ATLAS and CMS collaborations, Measurements of the Higgs boson production and decay rates and constraints on its couplings from a combined ATLAS and CMS analysis of the LHC pp collision data at $\sqrt{s}=7$ and $8 \mathrm{TeV}$, JHEP 08 (2016) 045 [arXiv: 1606.02266] [INSPIRE].

[8] S.L. Glashow, Partial-symmetries of weak interactions, Nucl. Phys. 22 (1961) 579 [InSPIRE].

[9] S. Weinberg, A model of leptons, Phys. Rev. Lett. 19 (1967) 1264 [INSPIRE].

[10] A. Salam, Weak and electromagnetic interactions, in Elementary particle theory, N. Svartholm ed., Almquist \& Wiksell, Sweden, (1968), pg. 367 [INSPIRE].

[11] F. Englert and R. Brout, Broken symmetry and the mass of gauge vector mesons, Phys. Rev. Lett. 13 (1964) 321 [INSPIRE].

[12] P.W. Higgs, Broken symmetries, massless particles and gauge fields, Phys. Lett. 12 (1964) 132 [INSPIRE].

[13] P.W. Higgs, Broken symmetries and the masses of gauge bosons, Phys. Rev. Lett. 13 (1964) 508 [INSPIRE]. 
[14] G.S. Guralnik, C.R. Hagen and T.W.B. Kibble, Global conservation laws and massless particles, Phys. Rev. Lett. 13 (1964) 585 [InSPIRE].

[15] P.W. Higgs, Spontaneous symmetry breakdown without massless bosons, Phys. Rev. 145 (1966) 1156 [INSPIRE].

[16] T.W.B. Kibble, Symmetry breaking in non-Abelian gauge theories, Phys. Rev. 155 (1967) 1554 [INSPIRE].

[17] H. Goldberg, Constraint on the photino mass from cosmology, Phys. Rev. Lett. 50 (1983) 1419 [Erratum ibid. 103 (2009) 099905] [INSPIRE].

[18] J.R. Ellis, J.S. Hagelin, D.V. Nanopoulos, K.A. Olive and M. Srednicki, Supersymmetric relics from the big bang, Nucl. Phys. B 238 (1984) 453 [INSPIRE].

[19] Particle Data Group collaboration, M. Tanabashi et al., Review of particle physics, Phys. Rev. D 98 (2018) 030001 [INSPIRE].

[20] Super-Kamiokande collaboration, Y. Fukuda et al., Evidence for oscillation of atmospheric neutrinos, Phys. Rev. Lett. 81 (1998) 1562 [hep-ex/9807003] [INSPIRE].

[21] J.F. Gunion, H.E. Haber, G.L. Kane and S. Dawson, The Higgs hunter's guide, Front. Phys. 80 (2000) 1, Perseus Books, U.S.A., (2000) [InSPIRE].

[22] G.C. Branco, P.M. Ferreira, L. Lavoura, M.N. Rebelo, M. Sher and J.P. Silva, Theory and phenomenology of two-Higgs-doublet models, Phys. Rept. 516 (2012) 1 [arXiv:1106.0034] [INSPIRE].

[23] E.A. Paschos, Diagonal neutral currents, Phys. Rev. D 15 (1977) 1966 [InSPIRE].

[24] S.L. Glashow and S. Weinberg, Natural conservation laws for neutral currents, Phys. Rev. D 15 (1977) 1958 [INSPIRE].

[25] W. Altmannshofer, S. Gori, A.L. Kagan, L. Silvestrini and J. Zupan, Uncovering mass generation through Higgs flavor violation, Phys. Rev. D 93 (2016) 031301 [arXiv: 1507.07927] [INSPIRE].

[26] W. Altmannshofer, J. Eby, S. Gori, M. Lotito, M. Martone and D. Tuckler, Collider signatures of flavorful Higgs bosons, Phys. Rev. D 94 (2016) 115032 [arXiv:1610.02398] [INSPIRE].

[27] LEP, DELPHI, OPAL, ALEPH and L3 collaborations, G. Abbiendi et al., Search for charged Higgs bosons: combined results using LEP data, Eur. Phys. J. C 73 (2013) 2463 [arXiv: 1301.6065] [INSPIRE].

[28] CDF collaboration, A. Abulencia et al., Search for charged Higgs bosons from top quark decays in py collisions at $\sqrt{s}=1.96 \mathrm{TeV}$., Phys. Rev. Lett. 96 (2006) 042003 [hep-ex/0510065] [INSPIRE].

[29] D0 collaboration, V.M. Abazov et al., Search for charged Higgs bosons in decays of top quarks, Phys. Rev. D 80 (2009) 051107 [arXiv:0906.5326] [INSPIRE].

[30] CDF collaboration, T.A. Aaltonen et al., Study of top-quark production and decays involving a tau lepton at CDF and limits on a charged-Higgs boson contribution, Phys. Rev. D 89 (2014) 091101 [arXiv:1402.6728] [inSPIRE].

[31] CMS collaboration, Search for a light charged Higgs boson in top quark decays in pp collisions at $\sqrt{s}=7 \mathrm{TeV}$, JHEP 07 (2012) 143 [arXiv:1205.5736] [INSPIRE]. 
[32] ATLAS collaboration, Search for charged Higgs bosons decaying via $H^{ \pm} \rightarrow \tau^{ \pm} \nu$ in fully hadronic final states using pp collision data at $\sqrt{s}=8 \mathrm{TeV}$ with the ATLAS detector, JHEP 03 (2015) 088 [arXiv: 1412.6663] [INSPIRE].

[33] ATLAS collaboration, Search for charged Higgs bosons decaying via $H^{+} \rightarrow \tau \nu$ in top quark pair events using pp collision data at $\sqrt{s}=7 \mathrm{TeV}$ with the ATLAS detector, JHEP 06 (2012) 039 [arXiv: 1204.2760] [INSPIRE].

[34] ATLAS collaboration, Search for charged Higgs bosons decaying via $H^{ \pm} \rightarrow \tau^{ \pm} \nu_{\tau}$ in the $\tau+$ jets and $\tau+$ lepton final states with $36 \mathrm{fb}^{-1}$ of pp collision data recorded at $\sqrt{\mathrm{s}}=13 \mathrm{TeV}$ with the ATLAS experiment, JHEP 09 (2018) 139 [arXiv:1807.07915] [INSPIRE].

[35] CDF collaboration, T. Aaltonen et al., Search for charged Higgs bosons in decays of top quarks in p $\bar{p}$ collisions at $\sqrt{s}=1.96$ TeV, Phys. Rev. Lett. 103 (2009) 101803 [arXiv:0907.1269] [INSPIRE].

[36] D0 collaboration, V.M. Abazov et al., Search for charged Higgs bosons in top quark decays, Phys. Lett. B 682 (2009) 278 [arXiv:0908.1811] [InSPIRE].

[37] ATLAS collaboration, Search for a light charged Higgs boson in the decay channel $H^{+} \rightarrow c \bar{s}$ in $t \bar{t}$ events using pp collisions at $\sqrt{s}=7 \mathrm{TeV}$ with the ATLAS detector, Eur. Phys. J. C $\mathbf{7 3}$ (2013) 2465 [arXiv: 1302.3694] [INSPIRE].

[38] CMS collaboration, Search for a light charged Higgs boson decaying to c $\bar{s}$ in pp collisions at $\sqrt{s}=8 \mathrm{TeV}$, JHEP $12(2015) 178$ [arXiv:1510.04252] [INSPIRE].

[39] CDF collaboration, T. Aaltonen et al., Search for a very light CP-odd Higgs boson in top

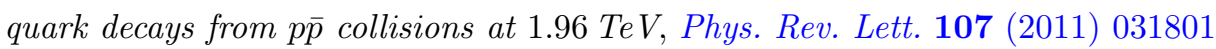
[arXiv:1104.5701] [INSPIRE].

[40] M. Aoki, S. Kanemura, K. Tsumura and K. Yagyu, Models of Yukawa interaction in the two Higgs doublet model and their collider phenomenology, Phys. Rev. D 80 (2009) 015017 [arXiv: 0902.4665] [INSPIRE].

[41] H.E. Logan and D. MacLennan, Charged Higgs phenomenology in the flipped two Higgs doublet model, Phys. Rev. D 81 (2010) 075016 [arXiv:1002.4916] [INSPIRE].

[42] A.G. Akeroyd, S. Moretti and J. Hernandez-Sanchez, Light charged Higgs bosons decaying to charm and bottom quarks in models with two or more Higgs doublets, Phys. Rev. D 85 (2012) 115002 [arXiv:1203.5769] [INSPIRE].

[43] Y. Grossman, Phenomenology of models with more than two Higgs doublets, Nucl. Phys. B 426 (1994) 355 [hep-ph/9401311] [INSPIRE].

[44] A.G. Akeroyd and W.J. Stirling, Light charged Higgs scalars at high-energy $e^{+} e^{-}$colliders, Nucl. Phys. B 447 (1995) 3 [InSPIRE].

[45] J. Alwall et al., The automated computation of tree-level and next-to-leading order differential cross sections and their matching to parton shower simulations, JHEP 07 (2014) 079 [arXiv: 1405.0301] [INSPIRE].

[46] J. Pumplin, D.R. Stump, J. Huston, H.L. Lai, P.M. Nadolsky and W.K. Tung, New generation of parton distributions with uncertainties from global QCD analysis, JHEP 07 (2002) 012 [hep-ph/0201195] [INSPIRE].

[47] M. Czakon and A. Mitov, Top++: a program for the calculation of the top-pair cross-section at hadron colliders, Comput. Phys. Commun. 185 (2014) 2930 [arXiv:1112.5675] [INSPIRE]. 
[48] S. Alekhin et al., The PDF4LHC working group interim report, arXiv:1101.0536 [INSPIRE].

[49] M. Botje et al., The PDF4LHC working group interim recommendations, arXiv:1101.0538 [INSPIRE].

[50] A.D. Martin, W.J. Stirling, R.S. Thorne and G. Watt, Uncertainties on $\alpha_{s}$ in global PDF analyses and implications for predicted hadronic cross sections, Eur. Phys. J. C 64 (2009) 653 [arXiv: 0905.3531] [INSPIRE].

[51] J. Gao et al., CT10 next-to-next-to-leading order global analysis of QCD, Phys. Rev. D 89 (2014) 033009 [arXiv: 1302.6246] [inSPIRE].

[52] R.D. Ball et al., Parton distributions with LHC data, Nucl. Phys. B 867 (2013) 244 [arXiv: 1207.1303] [INSPIRE].

[53] CMS collaboration, Measurement of the differential cross section for top quark pair production in pp collisions at $\sqrt{s}=8 \mathrm{TeV}$, Eur. Phys. J. C 75 (2015) 542 [arXiv: 1505.04480] [INSPIRE].

[54] Y. Li and F. Petriello, Combining QCD and electroweak corrections to dilepton production in FEWZ, Phys. Rev. D 86 (2012) 094034 [arXiv: 1208.5967] [INSPIRE].

[55] R. Gavin, Y. Li, F. Petriello and S. Quackenbush, W physics at the LHC with FEWZ 2.1, Comput. Phys. Commun. 184 (2013) 208 [arXiv:1201.5896] [INSPIRE].

[56] J.M. Campbell and R.K. Ellis, $t \bar{t} W^{ \pm}$production and decay at NLO, JHEP 07 (2012) 052 [arXiv: 1204.5678] [INSPIRE].

[57] M.V. Garzelli, A. Kardos, C.G. Papadopoulos and Z. Trócsányi, $t \bar{t} W^{ \pm}$and $t \bar{t} Z$ hadroproduction at $N L O$ accuracy in QCD with parton shower and hadronization effects, JHEP 11 (2012) 056 [arXiv: 1208.2665] [INSPIRE].

[58] S. Alioli, P. Nason, C. Oleari and E. Re, A general framework for implementing NLO calculations in shower Monte Carlo programs: the POWHEG BOX, JHEP 06 (2010) 043 [arXiv: 1002.2581] [INSPIRE].

[59] P. Nason, A new method for combining NLO QCD with shower Monte Carlo algorithms, JHEP 11 (2004) 040 [hep-ph/0409146] [INSPIRE].

[60] S. Frixione, P. Nason and C. Oleari, Matching NLO QCD computations with parton shower simulations: the POWHEG method, JHEP 11 (2007) 070 [arXiv:0709.2092] [INSPIRE].

[61] S. Alioli, P. Nason, C. Oleari and E. Re, NLO single-top production matched with shower in POWHEG: s- and t-channel contributions, JHEP 09 (2009) 111 [Erratum ibid. 02 (2010) 011] [arXiv:0907.4076] [INSPIRE].

[62] M. Aliev, H. Lacker, U. Langenfeld, S. Moch, P. Uwer and M. Wiedermann, HATHOR: HAdronic Top and Heavy quarks crOss section calculatoR, Comput. Phys. Commun. 182 (2011) 1034 [arXiv: 1007.1327] [inSPIRE].

[63] P. Kant et al., HatHor for single top-quark production: updated predictions and uncertainty estimates for single top-quark production in hadronic collisions, Comput. Phys. Commun. 191 (2015) 74 [arXiv: 1406.4403] [INSPIRE].

[64] T. Sjöstrand, S. Mrenna and P.Z. Skands, PYTHIA 6.4 physics and manual, JHEP 05 (2006) 026 [hep-ph/0603175] [INSPIRE].

[65] J.M. Campbell, R.K. Ellis and C. Williams, Vector boson pair production at the LHC, JHEP 07 (2011) 018 [arXiv: 1105.0020] [INSPIRE]. 
[66] LHC Higgs Cross Section Working Group collaboration, J.R. Andersen et al., Handbook of LHC Higgs cross sections: 3. Higgs properties, arXiv:1307.1347 [INSPIRE].

[67] GEANT4 collaboration, S. Agostinelli et al., GEANT4: a simulation toolkit, Nucl. Instrum. Meth. A 506 (2003) 250 [INSPIRE].

[68] CMS collaboration, Study of the underlying event at forward rapidity in pp collisions at $\sqrt{s}=0.9,2.76$ and $7 \mathrm{TeV}$, JHEP 04 (2013) 072 [arXiv: 1302.2394] [INSPIRE].

[69] CMS collaboration, Event generator tunes obtained from underlying event and multiparton scattering measurements, Eur. Phys. J. C 76 (2016) 155 [arXiv:1512.00815] [INSPIRE].

[70] CMS collaboration, The CMS experiment at the CERN LHC, 2008 JINST 3 S08004 [INSPIRE].

[71] CMS collaboration, Particle-flow reconstruction and global event description with the CMS detector, 2017 JINST 12 P10003 [arXiv:1706.04965] [INSPIRE].

[72] CMS collaboration, Energy calibration and resolution of the CMS electromagnetic calorimeter in pp collisions at $\sqrt{s}=7 \mathrm{TeV}, 2013$ JINST 8 P09009 [arXiv:1306.2016] [INSPIRE].

[73] CMS collaboration, Performance of photon reconstruction and identification with the CMS detector in proton-proton collisions at $\sqrt{s}=8 \mathrm{TeV}, 2015$ JINST $10 \mathrm{P} 08010$ [arXiv: 1502.02702] [INSPIRE].

[74] CMS collaboration, Performance of electron reconstruction and selection with the CMS detector in proton-proton collisions at $\sqrt{s}=8 \mathrm{TeV}, 2015$ JINST $10 \mathrm{P} 06005$ [arXiv: 1502.02701] [INSPIRE].

[75] M. Cacciari, G.P. Salam and G. Soyez, The anti- $k_{t}$ jet clustering algorithm, JHEP 04 (2008) 063 [arXiv: 0802.1189] [INSPIRE].

[76] M. Cacciari, G.P. Salam and G. Soyez, FastJet user manual, Eur. Phys. J. C 72 (2012) 1896 [arXiv: 1111.6097] [INSPIRE].

[77] CMS collaboration, Jet energy scale and resolution in the CMS experiment in pp collisions at $8 \mathrm{TeV}, 2017$ JINST $12 \mathrm{P} 02014$ [arXiv: 1607.03663] [INSPIRE].

[78] CMS collaboration, Determination of jet energy calibration and transverse momentum resolution in CMS, 2011 JINST 6 P11002 [arXiv:1107.4277] [INSPIRE].

[79] CMS collaboration, The CMS trigger system, 2017 JINST 12 P01020 [arXiv:1609. 02366] [INSPIRE].

[80] CMS collaboration, Identification of b-quark jets with the CMS experiment, 2013 JINST 8 P04013 [arXiv: 1211.4462] [INSPIRE].

[81] CMS collaboration, Measurement of the cross section ratio $\sigma_{t \bar{t} \bar{b} \bar{b}} / \sigma_{t \bar{t} j j}$ in pp collisions at $\sqrt{s}=8$ TeV, Phys. Lett. B 746 (2015) 132 [arXiv:1411.5621] [InSPIRE].

[82] CDF collaboration, A. Abulencia et al., Top quark mass measurement using the template method in the lepton + jets channel at CDF II, Phys. Rev. D 73 (2006) 032003 [hep-ex/0510048] [INSPIRE].

[83] CMS collaboration, Measurement of the top quark mass using proton-proton data at $\sqrt{s}=7$ and 8 TeV, Phys. Rev. D 93 (2016) 072004 [arXiv:1509.04044] [INSPIRE].

[84] CMS collaboration, CMS luminosity based on pixel cluster counting - Summer 2013 update, CMS-PAS-LUM-13-001, CERN, Geneva, Switzerland, (2013). 
[85] L. Moneta et al., The RooStats project, PoS (ACAT2010) 057, (2010) [arXiv:1009.1003] [INSPIRE].

[86] T. Junk, Confidence level computation for combining searches with small statistics, Nucl. Instrum. Meth. A 434 (1999) 435 [hep-ex/9902006] [INSPIRE].

[87] A.L. Read, Presentation of search results: the $C L_{s}$ technique, J. Phys. G 28 (2002) 2693 [INSPIRE].

[88] ATLAS and CMS collaborations and the LHC Higgs Combination Group, Procedure for the LHC Higgs boson search combination in Summer 2011, CMS-NOTE-2011-005, CERN, Geneva, Switzerland, (2011) [ATL-PHYS-PUB-2011-11].

[89] G. Cowan, K. Cranmer, E. Gross and O. Vitells, Asymptotic formulae for likelihood-based tests of new physics, Eur. Phys. J. C 71 (2011) 1554 [Erratum ibid. C 73 (2013) 2501] [arXiv:1007.1727] [INSPIRE]. 


\section{The CMS collaboration}

\section{Yerevan Physics Institute, Yerevan, Armenia}

A.M. Sirunyan, A. Tumasyan

\section{Institut für Hochenergiephysik, Wien, Austria}

W. Adam, F. Ambrogi, E. Asilar, T. Bergauer, J. Brandstetter, E. Brondolin, M. Dragicevic, J. Erö, A. Escalante Del Valle, M. Flechl, R. Frühwirth ${ }^{1}$, V.M. Ghete, J. Hrubec, M. Jeitler ${ }^{1}$, N. Krammer, I. Krätschmer, D. Liko, T. Madlener, I. Mikulec, N. Rad, H. Rohringer, J. Schieck ${ }^{1}$, R. Schöfbeck, M. Spanring, D. Spitzbart, A. Taurok, W. Waltenberger, J. Wittmann, C.-E. Wulz ${ }^{1}$, M. Zarucki

Institute for Nuclear Problems, Minsk, Belarus

V. Chekhovsky, V. Mossolov, J. Suarez Gonzalez

Universiteit Antwerpen, Antwerpen, Belgium

E.A. De Wolf, D. Di Croce, X. Janssen, J. Lauwers, M. Pieters, M. Van De Klundert, H. Van Haevermaet, P. Van Mechelen, N. Van Remortel

Vrije Universiteit Brussel, Brussel, Belgium

S. Abu Zeid, F. Blekman, J. D'Hondt, I. De Bruyn, J. De Clercq, K. Deroover, G. Flouris, D. Lontkovskyi, S. Lowette, I. Marchesini, S. Moortgat, L. Moreels, Q. Python, K. Skovpen, S. Tavernier, W. Van Doninck, P. Van Mulders, I. Van Parijs

Université Libre de Bruxelles, Bruxelles, Belgium

D. Beghin, B. Bilin, H. Brun, B. Clerbaux, G. De Lentdecker, H. Delannoy, B. Dorney, G. Fasanella, L. Favart, R. Goldouzian, A. Grebenyuk, A.K. Kalsi, T. Lenzi, J. Luetic, N. Postiau, E. Starling, L. Thomas, C. Vander Velde, P. Vanlaer, D. Vannerom, Q. Wang

\section{Ghent University, Ghent, Belgium}

T. Cornelis, D. Dobur, A. Fagot, M. Gul, I. Khvastunov², D. Poyraz, C. Roskas, D. Trocino, M. Tytgat, W. Verbeke, B. Vermassen, M. Vit, N. Zaganidis

\section{Université Catholique de Louvain, Louvain-la-Neuve, Belgium}

H. Bakhshiansohi, O. Bondu, S. Brochet, G. Bruno, C. Caputo, P. David, C. Delaere, M. Delcourt, B. Francois, A. Giammanco, G. Krintiras, V. Lemaitre, A. Magitteri, A. Mertens, M. Musich, K. Piotrzkowski, A. Saggio, M. Vidal Marono, S. Wertz, J. Zobec

\section{Centro Brasileiro de Pesquisas Fisicas, Rio de Janeiro, Brazil}

F.L. Alves, G.A. Alves, L. Brito, G. Correia Silva, C. Hensel, A. Moraes, M.E. Pol, P. Rebello Teles

Universidade do Estado do Rio de Janeiro, Rio de Janeiro, Brazil

E. Belchior Batista Das Chagas, W. Carvalho, J. Chinellato ${ }^{3}$, E. Coelho, E.M. Da Costa, G.G. Da Silveira ${ }^{4}$, D. De Jesus Damiao, C. De Oliveira Martins, S. Fonseca De Souza, H. Malbouisson, D. Matos Figueiredo, M. Melo De Almeida, C. Mora Herrera, L. Mundim, H. Nogima, W.L. Prado Da Silva, L.J. Sanchez Rosas, A. Santoro, A. Sznajder, M. Thiel, E.J. Tonelli Manganote ${ }^{3}$, F. Torres Da Silva De Araujo, A. Vilela Pereira 
Universidade Estadual Paulista ${ }^{a}$, Universidade Federal do ABC ${ }^{b}$, São Paulo, Brazil

S. Ahuja ${ }^{a}$, C.A. Bernardes ${ }^{a}$, L. Calligaris $^{a}$, T.R. Fernandez Perez Tomei ${ }^{a}$, E.M. Gregores $^{b}{ }$ P.G. Mercadante ${ }^{b}$, S.F. Novaes ${ }^{a}$, SandraS. Padula ${ }^{a}$, D. Romero Abad $^{b}$

Institute for Nuclear Research and Nuclear Energy, Bulgarian Academy of Sciences, Sofia, Bulgaria

A. Aleksandrov, R. Hadjiiska, P. Iaydjiev, A. Marinov, M. Misheva, M. Rodozov,

M. Shopova, G. Sultanov

University of Sofia, Sofia, Bulgaria

A. Dimitrov, L. Litov, B. Pavlov, P. Petkov

Beihang University, Beijing, China

W. Fang ${ }^{5}$, X. Gao ${ }^{5}$, L. Yuan

Institute of High Energy Physics, Beijing, China

M. Ahmad, J.G. Bian, G.M. Chen, H.S. Chen, M. Chen, Y. Chen, C.H. Jiang, D. Leggat,

H. Liao, Z. Liu, F. Romeo, S.M. Shaheen, A. Spiezia, J. Tao, C. Wang, Z. Wang, E. Yazgan,

H. Zhang, J. Zhao

State Key Laboratory of Nuclear Physics and Technology, Peking University, Beijing, China

Y. Ban, G. Chen, A. Levin, J. Li, L. Li, Q. Li, Y. Mao, S.J. Qian, D. Wang, Z. Xu

Tsinghua University, Beijing, China

Y. Wang

Universidad de Los Andes, Bogota, Colombia

C. Avila, A. Cabrera, C.A. Carrillo Montoya, L.F. Chaparro Sierra, C. Florez,

C.F. González Hernández, M.A. Segura Delgado

University of Split, Faculty of Electrical Engineering, Mechanical Engineering and Naval Architecture, Split, Croatia

B. Courbon, N. Godinovic, D. Lelas, I. Puljak, T. Sculac

University of Split, Faculty of Science, Split, Croatia

Z. Antunovic, M. Kovac

Institute Rudjer Boskovic, Zagreb, Croatia

V. Brigljevic, D. Ferencek, K. Kadija, B. Mesic, A. Starodumov ${ }^{6}$, T. Susa

University of Cyprus, Nicosia, Cyprus

M.W. Ather, A. Attikis, G. Mavromanolakis, J. Mousa, C. Nicolaou, F. Ptochos, P.A. Razis, H. Rykaczewski

Charles University, Prague, Czech Republic

M. Finger ${ }^{7}$, M. Finger Jr. ${ }^{7}$ 
Escuela Politecnica Nacional, Quito, Ecuador

E. Ayala

Universidad San Francisco de Quito, Quito, Ecuador

E. Carrera Jarrin

Academy of Scientific Research and Technology of the Arab Republic of Egypt, Egyptian Network of High Energy Physics, Cairo, Egypt

H. Abdalla ${ }^{8}$, A.A. Abdelalim ${ }^{9,10}$, A. Mohamed $^{10}$

National Institute of Chemical Physics and Biophysics, Tallinn, Estonia

S. Bhowmik, A. Carvalho Antunes De Oliveira, R.K. Dewanjee, K. Ehataht, M. Kadastik, M. Raidal, C. Veelken

Department of Physics, University of Helsinki, Helsinki, Finland

P. Eerola, H. Kirschenmann, J. Pekkanen, M. Voutilainen

Helsinki Institute of Physics, Helsinki, Finland

J. Havukainen, J.K. Heikkilä, T. Järvinen, V. Karimäki, R. Kinnunen, T. Lampén, K. Lassila-Perini, S. Laurila, S. Lehti, T. Lindén, P. Luukka, T. Mäenpää, H. Siikonen, E. Tuominen, J. Tuominiemi

\section{Lappeenranta University of Technology, Lappeenranta, Finland}

T. Tuuva

IRFU, CEA, Université Paris-Saclay, Gif-sur-Yvette, France

M. Besancon, F. Couderc, M. Dejardin, D. Denegri, J.L. Faure, F. Ferri, S. Ganjour, A. Givernaud, P. Gras, G. Hamel de Monchenault, P. Jarry, C. Leloup, E. Locci, J. Malcles, G. Negro, J. Rander, A. Rosowsky, M.Ö. Sahin, M. Titov

Laboratoire Leprince-Ringuet, Ecole polytechnique, CNRS/IN2P3, Université Paris-Saclay, Palaiseau, France

A. Abdulsalam ${ }^{11}$, C. Amendola, I. Antropov, F. Beaudette, P. Busson, C. Charlot, R. Granier de Cassagnac, I. Kucher, S. Lisniak, A. Lobanov, J. Martin Blanco, M. Nguyen, C. Ochando, G. Ortona, P. Paganini, P. Pigard, R. Salerno, J.B. Sauvan, Y. Sirois, A.G. Stahl Leiton, A. Zabi, A. Zghiche

Université de Strasbourg, CNRS, IPHC UMR 7178, Strasbourg, France J.-L. Agram ${ }^{12}$, J. Andrea, D. Bloch, J.-M. Brom, E.C. Chabert, V. Cherepanov, C. Collard, E. Conte ${ }^{12}$, J.-C. Fontaine ${ }^{12}$, D. Gelé, U. Goerlach, M. Jansová, A.-C. Le Bihan, N. Tonon, P. Van Hove

Centre de Calcul de l'Institut National de Physique Nucleaire et de Physique des Particules, CNRS/IN2P3, Villeurbanne, France

S. Gadrat 
Université de Lyon, Université Claude Bernard Lyon 1, CNRS-IN2P3, Institut de Physique Nucléaire de Lyon, Villeurbanne, France

S. Beauceron, C. Bernet, G. Boudoul, N. Chanon, R. Chierici, D. Contardo, P. Depasse, H. El Mamouni, J. Fay, L. Finco, S. Gascon, M. Gouzevitch, G. Grenier, B. Ille, F. Lagarde, I.B. Laktineh, H. Lattaud, M. Lethuillier, L. Mirabito, A.L. Pequegnot, S. Perries, A. Popov ${ }^{13}$, V. Sordini, M. Vander Donckt, S. Viret, S. Zhang

\section{Georgian Technical University, Tbilisi, Georgia}

A. Khvedelidze ${ }^{7}$

Tbilisi State University, Tbilisi, Georgia

Z. Tsamalaidze ${ }^{7}$

RWTH Aachen University, I. Physikalisches Institut, Aachen, Germany

C. Autermann, L. Feld, M.K. Kiesel, K. Klein, M. Lipinski, M. Preuten, M.P. Rauch, C. Schomakers, J. Schulz, M. Teroerde, B. Wittmer, V. Zhukov ${ }^{13}$

RWTH Aachen University, III. Physikalisches Institut A, Aachen, Germany

A. Albert, D. Duchardt, M. Endres, M. Erdmann, T. Esch, R. Fischer, S. Ghosh, A. Güth,

T. Hebbeker, C. Heidemann, K. Hoepfner, H. Keller, S. Knutzen, L. Mastrolorenzo,

M. Merschmeyer, A. Meyer, P. Millet, S. Mukherjee, T. Pook, M. Radziej, H. Reithler,

M. Rieger, F. Scheuch, A. Schmidt, D. Teyssier

RWTH Aachen University, III. Physikalisches Institut B, Aachen, Germany

G. Flügge, O. Hlushchenko, B. Kargoll, T. Kress, A. Künsken, T. Müller, A. Nehrkorn, A. Nowack, C. Pistone, O. Pooth, H. Sert, A. Stahl ${ }^{14}$

Deutsches Elektronen-Synchrotron, Hamburg, Germany

M. Aldaya Martin, T. Arndt, C. Asawatangtrakuldee, I. Babounikau, K. Beernaert, O. Behnke, U. Behrens, A. Bermúdez Martínez, D. Bertsche, A.A. Bin Anuar, K. Borras ${ }^{15}$, V. Botta, A. Campbell, P. Connor, C. Contreras-Campana, F. Costanza, V. Danilov, A. De Wit, M.M. Defranchis, C. Diez Pardos, D. Domínguez Damiani, G. Eckerlin, T. Eichhorn, A. Elwood, E. Eren, E. Gallo ${ }^{16}$, A. Geiser, J.M. Grados Luyando, A. Grohsjean, P. Gunnellini, M. Guthoff, M. Haranko, A. Harb, J. Hauk, H. Jung, M. Kasemann, J. Keaveney, C. Kleinwort, J. Knolle, D. Krücker, W. Lange, A. Lelek, T. Lenz, K. Lipka, W. Lohmann ${ }^{17}$, R. Mankel, I.-A. Melzer-Pellmann, A.B. Meyer, M. Meyer, M. Missiroli, G. Mittag, J. Mnich, V. Myronenko, S.K. Pflitsch, D. Pitzl, A. Raspereza, M. Savitskyi, P. Saxena, P. Schütze, C. Schwanenberger, R. Shevchenko, A. Singh, N. Stefaniuk, H. Tholen, A. Vagnerini, G.P. Van Onsem, R. Walsh, Y. Wen, K. Wichmann, C. Wissing, O. Zenaiev

\section{University of Hamburg, Hamburg, Germany}

R. Aggleton, S. Bein, L. Benato, A. Benecke, V. Blobel, M. Centis Vignali, T. Dreyer, E. Garutti, D. Gonzalez, J. Haller, A. Hinzmann, M. Hoffmann, A. Karavdina, G. Kasieczka, R. Klanner, R. Kogler, N. Kovalchuk, S. Kurz, V. Kutzner, J. Lange, D. Marconi, J. Multhaup, M. Niedziela, D. Nowatschin, A. Perieanu, A. Reimers, O. Rieger, 
C. Scharf, P. Schleper, S. Schumann, J. Schwandt, J. Sonneveld, H. Stadie, G. Steinbrück, F.M. Stober, M. Stöver, D. Troendle, E. Usai, A. Vanhoefer, B. Vormwald

Karlsruher Institut fuer Technologie, Karlsruhe, Germany

M. Akbiyik, C. Barth, M. Baselga, S. Baur, E. Butz, R. Caspart, T. Chwalek, F. Colombo, W. De Boer, A. Dierlamm, N. Faltermann, B. Freund, M. Giffels, M.A. Harrendorf, F. Hartmann ${ }^{14}$, S.M. Heindl, U. Husemann, F. Kassel ${ }^{14}$, I. Katkov ${ }^{13}$, S. Kudella, H. Mildner, S. Mitra, M.U. Mozer, Th. Müller, M. Plagge, G. Quast, K. Rabbertz, M. Schröder, I. Shvetsov, G. Sieber, H.J. Simonis, R. Ulrich, S. Wayand, M. Weber, T. Weiler, S. Williamson, C. Wöhrmann, R. Wolf

Institute of Nuclear and Particle Physics (INPP), NCSR Demokritos, Aghia Paraskevi, Greece

G. Anagnostou, G. Daskalakis, T. Geralis, A. Kyriakis, D. Loukas, G. Paspalaki, I. TopsisGiotis

National and Kapodistrian University of Athens, Athens, Greece

G. Karathanasis, S. Kesisoglou, P. Kontaxakis, A. Panagiotou, N. Saoulidou, E. Tziaferi, K. Vellidis

National Technical University of Athens, Athens, Greece

K. Kousouris, I. Papakrivopoulos, G. Tsipolitis

University of Ioánnina, Ioánnina, Greece

I. Evangelou, C. Foudas, P. Gianneios, P. Katsoulis, P. Kokkas, S. Mallios, N. Manthos, I. Papadopoulos, E. Paradas, J. Strologas, F.A. Triantis, D. Tsitsonis

MTA-ELTE Lendület CMS Particle and Nuclear Physics Group, Eötvös Loránd University, Budapest, Hungary

M. Csanad, N. Filipovic, P. Major, M.I. Nagy, G. Pasztor, O. Surányi, G.I. Veres

Wigner Research Centre for Physics, Budapest, Hungary

G. Bencze, C. Hajdu, D. Horvath ${ }^{18}$, Á. Hunyadi, F. Sikler, T.Á. Vámi, V. Veszpremi, G. Vesztergombi ${ }^{\dagger}$

Institute of Nuclear Research ATOMKI, Debrecen, Hungary

N. Beni, S. Czellar, J. Karancsi ${ }^{20}$, A. Makovec, J. Molnar, Z. Szillasi

Institute of Physics, University of Debrecen, Debrecen, Hungary

M. Bartók ${ }^{19}$, P. Raics, Z.L. Trocsanyi, B. Ujvari

Indian Institute of Science (IISc), Bangalore, India

S. Choudhury, J.R. Komaragiri, P.C. Tiwari

National Institute of Science Education and Research, HBNI, Bhubaneswar, India

S. Bahinipati21 , C. Kar, P. Mal, K. Mandal, A. Nayak ${ }^{22}$, D.K. Sahoo ${ }^{21}$, S.K. Swain 
Panjab University, Chandigarh, India

S. Bansal, S.B. Beri, V. Bhatnagar, S. Chauhan, R. Chawla, N. Dhingra, R. Gupta,

A. Kaur, A. Kaur, M. Kaur, S. Kaur, R. Kumar, P. Kumari, M. Lohan, A. Mehta,

K. Sandeep, S. Sharma, J.B. Singh, G. Walia

University of Delhi, Delhi, India

A. Bhardwaj, B.C. Choudhary, R.B. Garg, M. Gola, S. Keshri, Ashok Kumar, S. Malhotra, M. Naimuddin, P. Priyanka, K. Ranjan, Aashaq Shah, R. Sharma

Saha Institute of Nuclear Physics, HBNI, Kolkata, India

R. Bhardwaj ${ }^{23}$, M. Bharti, R. Bhattacharya, S. Bhattacharya, U. Bhawandeep ${ }^{23}$, D. Bhowmik, S. Dey, S. Dutt ${ }^{23}$, S. Dutta, S. Ghosh, K. Mondal, S. Nandan, A. Purohit, P.K. Rout, A. Roy, S. Roy Chowdhury, S. Sarkar, M. Sharan, B. Singh, S. Thakur ${ }^{23}$

Indian Institute of Technology Madras, Madras, India

P.K. Behera

Bhabha Atomic Research Centre, Mumbai, India

R. Chudasama, D. Dutta, V. Jha, V. Kumar, P.K. Netrakanti, L.M. Pant, P. Shukla

Tata Institute of Fundamental Research-A, Mumbai, India

T. Aziz, M.A. Bhat, S. Dugad, G.B. Mohanty, N. Sur, B. Sutar, RavindraKumar Verma

Tata Institute of Fundamental Research-B, Mumbai, India

S. Banerjee, S. Bhattacharya, S. Chatterjee, P. Das, M. Guchait, Sa. Jain, S. Kumar, M. Maity ${ }^{24}$, G. Majumder, K. Mazumdar, N. Sahoo, T. Sarkar ${ }^{24}$

Indian Institute of Science Education and Research (IISER), Pune, India

S. Chauhan, S. Dube, V. Hegde, A. Kapoor, K. Kothekar, S. Pandey, A. Rane, S. Sharma

Institute for Research in Fundamental Sciences (IPM), Tehran, Iran

S. Chenarani ${ }^{25}$, E. Eskandari Tadavani, S.M. Etesami ${ }^{25}$, M. Khakzad, M. Mohammadi Najafabadi, M. Naseri, F. Rezaei Hosseinabadi, B. Safarzadeh ${ }^{26}$, M. Zeinali

University College Dublin, Dublin, Ireland

M. Felcini, M. Grunewald

INFN Sezione di Bari ${ }^{a}$, Università di Bari ${ }^{b}$, Politecnico di Bari ${ }^{c}$, Bari, Italy M. Abbrescia ${ }^{a, b}$, C. Calabria ${ }^{a, b}$, A. Colaleo ${ }^{a}$, D. Creanza ${ }^{a, c}$, L. Cristella ${ }^{a, b}$, N. De Filippis ${ }^{a, c}$, M. De Palma ${ }^{a, b}$, A. Di Florio ${ }^{a}, b$, F. Errico ${ }^{a, b}$, L. Fiore $^{a}$, A. Gelmi ${ }^{a, b}$,

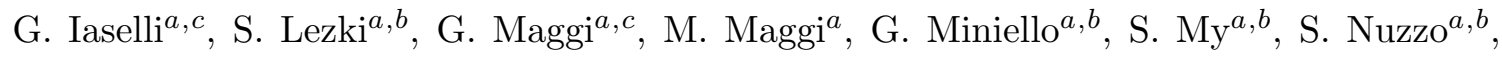
A. Pompili ${ }^{a, b}$, G. Pugliese ${ }^{a, c}$, R. Radogna ${ }^{a}$, A. Ranieri ${ }^{a}$, G. Selvaggi ${ }^{a, b}$, A. Sharma ${ }^{a}$, L. Silvestris ${ }^{a}, 14$, R. Venditti ${ }^{a}$, P. Verwilligen ${ }^{a}$, G. Zito ${ }^{a}$

INFN Sezione di Bologna ${ }^{a}$, Università di Bologna ${ }^{b}$, Bologna, Italy

G. Abbiendi ${ }^{a}$, C. Battilana ${ }^{a, b}$, D. Bonacorsi ${ }^{a, b}$, L. Borgonovi $^{a, b}$, S. Braibant-Giacomelli ${ }^{a, b}$, L. Brigliadori ${ }^{a, b}$, R. Campanini ${ }^{a, b}$, P. Capiluppi ${ }^{a, b}$, A. Castro ${ }^{a, b}$, F.R. Cavallo ${ }^{a}$, S.S. Chhibra ${ }^{a, b}$, C. Ciocca ${ }^{a}$, G. Codispoti ${ }^{a, b}$, M. Cuffiani ${ }^{a, b}$, G.M. Dallavalle $^{a}$, F. Fabbri ${ }^{a}$, A. Fanfani ${ }^{a, b}$, P. Giacomelli ${ }^{a}$, C. Grandi ${ }^{a}$, L. Guiducci ${ }^{a}, b$, S. Marcellini ${ }^{a}$, G. Masetti ${ }^{a}$, 


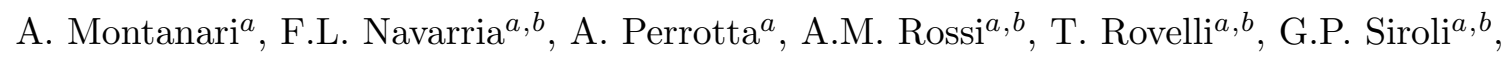
N. Tosi ${ }^{a}$

INFN Sezione di Catania ${ }^{a}$, Università di Catania ${ }^{b}$, Catania, Italy

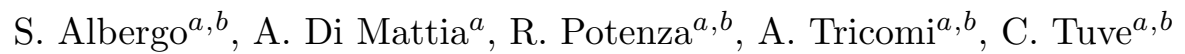

INFN Sezione di Firenze ${ }^{a}$, Università di Firenze ${ }^{b}$, Firenze, Italy

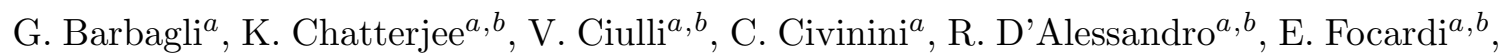
G. Latino, P. Lenzi ${ }^{a, b}$, M. Meschini ${ }^{a}$, S. Paoletti ${ }^{a}$, L. Russo $^{a, 27}$, G. Sguazzoni ${ }^{a}$, D. Strom ${ }^{a}$, L. Viliani ${ }^{a}$

INFN Laboratori Nazionali di Frascati, Frascati, Italy

L. Benussi, S. Bianco, F. Fabbri, D. Piccolo, F. Primavera ${ }^{14}$

INFN Sezione di Genova ${ }^{a}$, Università di Genova ${ }^{b}$, Genova, Italy

F. Ferro ${ }^{a}$, F. Ravera ${ }^{a, b}$, E. Robutti $^{a}$, S. Tosi ${ }^{a, b}$

INFN Sezione di Milano-Bicocca ${ }^{a}$, Università di Milano-Bicocca ${ }^{b}$, Milano, Italy

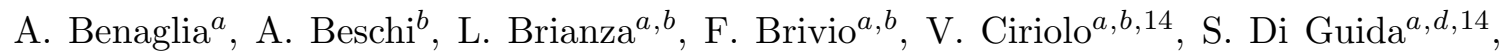

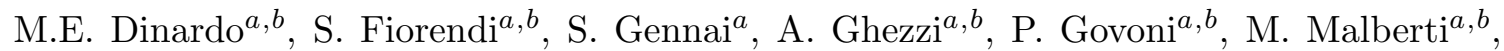
S. Malvezzi ${ }^{a}$, A. Massironi ${ }^{a, b}$, D. Menasce ${ }^{a}$, L. Moroni ${ }^{a}$, M. Paganoni ${ }^{a}, b$, D. Pedrini ${ }^{a}$, S. Ragazzi ${ }^{a, b}$, T. Tabarelli de Fatis ${ }^{a, b}$

INFN Sezione di Napoli ${ }^{a}$, Università di Napoli 'Federico II' ${ }^{b}$, Napoli, Italy, Università della Basilicata ${ }^{c}$, Potenza, Italy, Università G. Marconi ${ }^{d}$, Roma, Italy

S. Buontempo ${ }^{a}$, N. Cavallo ${ }^{a, c}$, A. Di Crescenzo ${ }^{a, b}$, F. Fabozzi ${ }^{a, c}$, F. Fienga ${ }^{a}$, G. Galati ${ }^{a}$, A.O.M. Iorio ${ }^{a, b}$, W.A. $\mathrm{Khan}^{a}$, L. Lista ${ }^{a}$, S. Meola ${ }^{a, d, 14}$, P. Paolucci ${ }^{a, 14}$, C. Sciacca ${ }^{a, b}$, E. Voevodina $a^{a, b}$

INFN Sezione di Padova ${ }^{a}$, Università di Padova ${ }^{b}$, Padova, Italy, Università di Trento ${ }^{c}$, Trento, Italy

P. Azzi ${ }^{a}$, N. Bacchetta ${ }^{a}$, D. Bisello ${ }^{a, b}$, A. Boletti ${ }^{a, b}$, A. Bragagnolo, R. Carlin ${ }^{a, b}$, P. Checchia ${ }^{a}$, M. Dall'Osso ${ }^{a}, b$, P. De Castro Manzano ${ }^{a}$, T. Dorigo ${ }^{a}$, U. Dosselli ${ }^{a}$, F. Gasparini ${ }^{a, b}$, U. Gasparini ${ }^{a, b}$, A. Gozzelino ${ }^{a}$, S. Lacaprara ${ }^{a}$, P. Lujan, M. Margoni ${ }^{a, b}$, A.T. Meneguzzo ${ }^{a, b}$, P. Ronchese ${ }^{a, b}$, R. Rossin ${ }^{a, b}$, F. Simonetto ${ }^{a, b}$, A. Tiko, E. Torassa ${ }^{a}$, M. Zanetti ${ }^{a, b}$, P. Zotto ${ }^{a, b}$, G. Zumerle ${ }^{a, b}$

INFN Sezione di Pavia ${ }^{a}$, Università di Pavia ${ }^{b}$, Pavia, Italy

A. Braghieri ${ }^{a}$, A. Magnani ${ }^{a}$, P. Montagna ${ }^{a, b}$, S.P. Ratti ${ }^{a}, b, V . \operatorname{Re}^{a}$, M. Ressegotti $^{a, b}$, C. Riccardi ${ }^{a, b}$, P. Salvini ${ }^{a}$, I. Vai ${ }^{a, b}$, P. Vitulo ${ }^{a, b}$

INFN Sezione di Perugia ${ }^{a}$, Università di Perugia ${ }^{b}$, Perugia, Italy

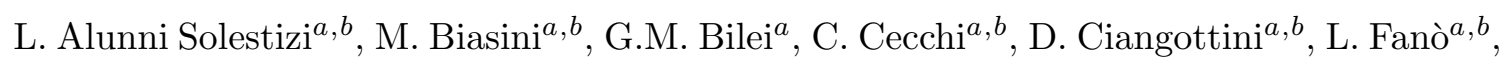
P. Lariccia ${ }^{a, b}$, E. Manoni ${ }^{a}$, G. Mantovani ${ }^{a, b}$, V. Mariani ${ }^{a, b}$, M. Menichelli ${ }^{a}$, A. Rossi ${ }^{a, b}$, A. Santocchia ${ }^{a, b}$, D. Spiga ${ }^{a}$ 
INFN Sezione di Pisa ${ }^{a}$, Università di Pisa ${ }^{b}$, Scuola Normale Superiore di Pisa ${ }^{c}$, Pisa, Italy

K. Androsov ${ }^{a}$, P. Azzurri ${ }^{a}$, G. Bagliesi ${ }^{a}$, L. Bianchini ${ }^{a}$, T. Boccali ${ }^{a}$, L. Borrello,

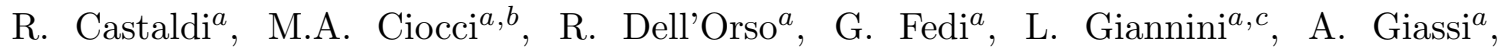
M.T. Grippo ${ }^{a}$, F. Ligabue ${ }^{a, c}$, E. Manca ${ }^{a, c}$, G. Mandorli ${ }^{a, c}$, A. Messineo ${ }^{a, b}$, F. Palla ${ }^{a}$,

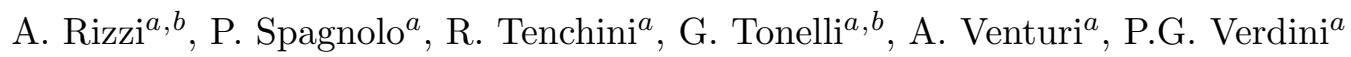

INFN Sezione di Roma ${ }^{a}$, Sapienza Università di Roma ${ }^{b}$, Rome, Italy

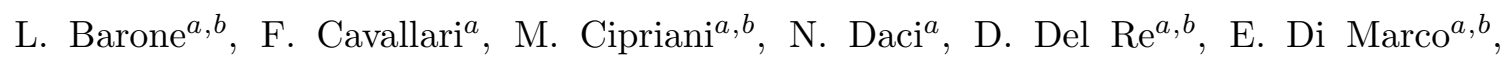
M. Diemoz ${ }^{a}$, S. Gelli ${ }^{a, b}$, E. Longo ${ }^{a, b}$, B. Marzocchi ${ }^{a, b}$, P. Meridiani $^{a}$, G. Organtini ${ }^{a, b}$,

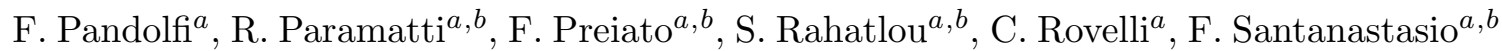

INFN Sezione di Torino ${ }^{a}$, Università di Torino ${ }^{b}$, Torino, Italy, Università del Piemonte Orientale ${ }^{c}$, Novara, Italy

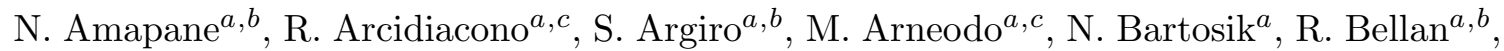

C. Biino ${ }^{a}$, N. Cartiglia $a$, F. Cenna ${ }^{a, b}$, S. Cometti, M. Costa ${ }^{a, b}$, R. Covarelli ${ }^{a, b}$,

N. Demaria ${ }^{a}$, B. Kiani ${ }^{a, b}$, C. Mariotti ${ }^{a}$, S. Maselli ${ }^{a}$, E. Migliore ${ }^{a, b}$, V. Monaco ${ }^{a, b}$,

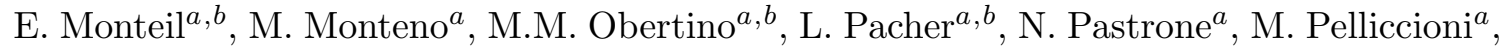
G.L. Pinna Angioni ${ }^{a, b}$, A. Romero ${ }^{a, b}$, M. Ruspa ${ }^{a, c}$, R. Sacchi ${ }^{a, b}$, K. Shchelina ${ }^{a, b}$, V. Sola ${ }^{a}$, A. Solano ${ }^{a, b}$, D. Soldi, A. Staiano ${ }^{a}$

INFN Sezione di Trieste ${ }^{a}$, Università di Trieste ${ }^{b}$, Trieste, Italy

S. Belforte ${ }^{a}$, V. Candelise ${ }^{a, b}$, M. Casarsa ${ }^{a}$, F. Cossutti ${ }^{a}$, G. Della Ricca ${ }^{a, b}$, F. Vazzoler $^{a}, b$, A. Zanetti ${ }^{a}$

Kyungpook National University, Daegu, Korea

D.H. Kim, G.N. Kim, M.S. Kim, J. Lee, S. Lee, S.W. Lee, C.S. Moon, Y.D. Oh, S. Sekmen, D.C. Son, Y.C. Yang

Chonnam National University, Institute for Universe and Elementary Particles, Kwangju, Korea

H. Kim, D.H. Moon, G. Oh

Hanyang University, Seoul, Korea

J. Goh, T.J. Kim

Korea University, Seoul, Korea

S. Cho, S. Choi, Y. Go, D. Gyun, S. Ha, B. Hong, Y. Jo, K. Lee, K.S. Lee, S. Lee, J. Lim, S.K. Park, Y. Roh

Sejong University, Seoul, Korea

H.S. Kim

\section{Seoul National University, Seoul, Korea}

M. Citron, J. Almond, Y.J. Jwa, J. Kim, J.S. Kim, H. Lee, K. Lee, K. Nam, B.H. Oh, S.B. Oh, B.C. Radburn-Smith, S.h. Seo, U.K. Yang, H.D. Yoo, G.B. Yu

University of Seoul, Seoul, Korea

D. Jeon, H. Kim, J.H. Kim, J.S.H. Lee, I.C. Park 
Sungkyunkwan University, Suwon, Korea

Y. Choi, C. Hwang, J. Lee, I. Yu

Vilnius University, Vilnius, Lithuania

V. Dudenas, A. Juodagalvis, J. Vaitkus

National Centre for Particle Physics, Universiti Malaya, Kuala Lumpur, Malaysia

I. Ahmed, Z.A. Ibrahim, M.A.B. Md Ali ${ }^{28}$, F. Mohamad Idris ${ }^{29}$, W.A.T. Wan Abdullah, M.N. Yusli, Z. Zolkapli

Centro de Investigacion y de Estudios Avanzados del IPN, Mexico City, Mexico

H. Castilla-Valdez, E. De La Cruz-Burelo, M.C. Duran-Osuna, I. Heredia-De La Cruz ${ }^{30}$, R. Lopez-Fernandez, J. Mejia Guisao, R.I. Rabadan-Trejo, G. Ramirez-Sanchez, R ReyesAlmanza, A. Sanchez-Hernandez

Universidad Iberoamericana, Mexico City, Mexico

S. Carrillo Moreno, C. Oropeza Barrera, F. Vazquez Valencia

Benemerita Universidad Autonoma de Puebla, Puebla, Mexico

J. Eysermans, I. Pedraza, H.A. Salazar Ibarguen, C. Uribe Estrada

Universidad Autónoma de San Luis Potosí, San Luis Potosí, Mexico

A. Morelos Pineda

University of Auckland, Auckland, New Zealand

D. Krofcheck

University of Canterbury, Christchurch, New Zealand

S. Bheesette, P.H. Butler

National Centre for Physics, Quaid-I-Azam University, Islamabad, Pakistan

A. Ahmad, M. Ahmad, M.I. Asghar, Q. Hassan, H.R. Hoorani, A. Saddique, M.A. Shah, M. Shoaib, M. Waqas

National Centre for Nuclear Research, Swierk, Poland

H. Bialkowska, M. Bluj, B. Boimska, T. Frueboes, M. Górski, M. Kazana, K. Nawrocki, M. Szleper, P. Traczyk, P. Zalewski

Institute of Experimental Physics, Faculty of Physics, University of Warsaw, Warsaw, Poland

K. Bunkowski, A. Byszuk ${ }^{31}$, K. Doroba, A. Kalinowski, M. Konecki, J. Krolikowski, M. Misiura, M. Olszewski, A. Pyskir, M. Walczak

Laboratório de Instrumentação e Física Experimental de Partículas, Lisboa, Portugal

P. Bargassa, C. Beirão Da Cruz E Silva, A. Di Francesco, P. Faccioli, B. Galinhas, M. Gallinaro, J. Hollar, N. Leonardo, L. Lloret Iglesias, M.V. Nemallapudi, J. Seixas, G. Strong, O. Toldaiev, D. Vadruccio, J. Varela 
Joint Institute for Nuclear Research, Dubna, Russia

S. Afanasiev, V. Alexakhin, P. Bunin, M. Gavrilenko, A. Golunov, I. Golutvin, N. Gorbounov, V. Karjavin, A. Lanev, A. Malakhov, V. Matveev ${ }^{32,33}$, P. Moisenz, V. Palichik, V. Perelygin, M. Savina, S. Shmatov, V. Smirnov, N. Voytishin, A. Zarubin

Petersburg Nuclear Physics Institute, Gatchina (St. Petersburg), Russia

V. Golovtsov, Y. Ivanov, V. Kim ${ }^{34}$, E. Kuznetsova ${ }^{35}$, P. Levchenko, V. Murzin, V. Oreshkin, I. Smirnov, D. Sosnov, V. Sulimov, L. Uvarov, S. Vavilov, A. Vorobyev

Institute for Nuclear Research, Moscow, Russia

Yu. Andreev, A. Dermenev, S. Gninenko, N. Golubev, A. Karneyeu, M. Kirsanov, N. Krasnikov, A. Pashenkov, D. Tlisov, A. Toropin

Institute for Theoretical and Experimental Physics, Moscow, Russia

V. Epshteyn, V. Gavrilov, N. Lychkovskaya, V. Popov, I. Pozdnyakov, G. Safronov, A. Spiridonov, A. Stepennov, V. Stolin, M. Toms, E. Vlasov, A. Zhokin

Moscow Institute of Physics and Technology, Moscow, Russia

T. Aushev, A. Bylinkin ${ }^{33}$

National Research Nuclear University 'Moscow Engineering Physics Institute' (MEPhI), Moscow, Russia

R. Chistov ${ }^{36}$, M. Danilov ${ }^{36}$, P. Parygin, D. Philippov, S. Polikarpov ${ }^{36}$, E. Tarkovskii

P.N. Lebedev Physical Institute, Moscow, Russia

V. Andreev, M. Azarkin ${ }^{33}$, I. Dremin ${ }^{33}$, M. Kirakosyan ${ }^{33}$, S.V. Rusakov, A. Terkulov

Skobeltsyn Institute of Nuclear Physics, Lomonosov Moscow State University, Moscow, Russia

A. Baskakov, A. Belyaev, E. Boos, V. Bunichev, M. Dubinin ${ }^{37}$, L. Dudko, A. Gribushin, V. Klyukhin, O. Kodolova, I. Lokhtin, I. Miagkov, S. Obraztsov, M. Perfilov, S. Petrushanko, V. Savrin

Novosibirsk State University (NSU), Novosibirsk, Russia

V. Blinov ${ }^{38}$, T. Dimova ${ }^{38}$, L. Kardapoltsev ${ }^{38}$, D. Shtol ${ }^{38}$, Y. Skovpen ${ }^{38}$

Institute for High Energy Physics of National Research Centre 'Kurchatov Institute', Protvino, Russia

I. Azhgirey, I. Bayshev, S. Bitioukov, D. Elumakhov, A. Godizov, V. Kachanov, A. Kalinin, D. Konstantinov, P. Mandrik, V. Petrov, R. Ryutin, S. Slabospitskii, A. Sobol, S. Troshin,

N. Tyurin, A. Uzunian, A. Volkov

National Research Tomsk Polytechnic University, Tomsk, Russia

A. Babaev, S. Baidali

University of Belgrade, Faculty of Physics and Vinca Institute of Nuclear Sciences, Belgrade, Serbia

P. Adzic ${ }^{39}$, P. Cirkovic, D. Devetak, M. Dordevic, J. Milosevic 
Centro de Investigaciones Energéticas Medioambientales y Tecnológicas (CIEMAT), Madrid, Spain

J. Alcaraz Maestre, A. Álvarez Fernández, I. Bachiller, M. Barrio Luna, J.A. Brochero Cifuentes, M. Cerrada, N. Colino, B. De La Cruz, A. Delgado Peris, C. Fernandez Bedoya, J.P. Fernández Ramos, J. Flix, M.C. Fouz, O. Gonzalez Lopez, S. Goy Lopez, J.M. Hernandez, M.I. Josa, D. Moran, A. Pérez-Calero Yzquierdo, J. Puerta Pelayo, I. Redondo, L. Romero, M.S. Soares, A. Triossi

\section{Universidad Autónoma de Madrid, Madrid, Spain}

C. Albajar, J.F. de Trocóniz

\section{Universidad de Oviedo, Oviedo, Spain}

J. Cuevas, C. Erice, J. Fernandez Menendez, S. Folgueras, I. Gonzalez Caballero, J.R. González Fernández, E. Palencia Cortezon, V. Rodríguez Bouza, S. Sanchez Cruz, P. Vischia, J.M. Vizan Garcia

Instituto de Física de Cantabria (IFCA), CSIC-Universidad de Cantabria, Santander, Spain

I.J. Cabrillo, A. Calderon, B. Chazin Quero, J. Duarte Campderros, M. Fernandez, P.J. Fernández Manteca, A. García Alonso, J. Garcia-Ferrero, G. Gomez, A. Lopez Virto, J. Marco, C. Martinez Rivero, P. Martinez Ruiz del Arbol, F. Matorras, J. Piedra Gomez, C. Prieels, T. Rodrigo, A. Ruiz-Jimeno, L. Scodellaro, N. Trevisani, I. Vila, R. Vilar Cortabitarte

\section{CERN, European Organization for Nuclear Research, Geneva, Switzerland}

D. Abbaneo, B. Akgun, E. Auffray, P. Baillon, A.H. Ball, D. Barney, J. Bendavid, M. Bianco, A. Bocci, C. Botta, T. Camporesi, M. Cepeda, G. Cerminara, E. Chapon, Y. Chen, G. Cucciati, D. d'Enterria, A. Dabrowski, V. Daponte, A. David, A. De Roeck, N. Deelen, M. Dobson, T. du Pree, M. Dünser, N. Dupont, A. Elliott-Peisert, P. Everaerts, F. Fallavollita ${ }^{40}$, D. Fasanella, G. Franzoni, J. Fulcher, W. Funk, D. Gigi, A. Gilbert, K. Gill, F. Glege, D. Gulhan, J. Hegeman, V. Innocente, A. Jafari, P. Janot, O. Karacheban ${ }^{17}$, J. Kieseler, A. Kornmayer, M. Krammer ${ }^{1}$, C. Lange, P. Lecoq, C. Lourenço, L. Malgeri, M. Mannelli, F. Meijers, J.A. Merlin, S. Mersi, E. Meschi, P. Milenovic ${ }^{41}$, F. Moortgat, M. Mulders, J. Ngadiuba, S. Orfanelli, L. Orsini, F. Pantaleo ${ }^{14}$, L. Pape, E. Perez, M. Peruzzi, A. Petrilli, G. Petrucciani, A. Pfeiffer, M. Pierini, F.M. Pitters, D. Rabady, A. Racz, T. Reis, G. Rolandi ${ }^{42}$, M. Rovere, H. Sakulin, C. Schäfer, C. Schwick, M. Seidel, M. Selvaggi, A. Sharma, P. Silva, P. Sphicas ${ }^{43}$, A. Stakia, J. Steggemann, M. Tosi, D. Treille, A. Tsirou, V. Veckalns ${ }^{44}$, W.D. Zeuner

\section{Paul Scherrer Institut, Villigen, Switzerland}

W. Bertl ${ }^{\dagger}$, L. Caminada ${ }^{45}$, K. Deiters, W. Erdmann, R. Horisberger, Q. Ingram, H.C. Kaestli, D. Kotlinski, U. Langenegger, T. Rohe, S.A. Wiederkehr 
ETH Zurich - Institute for Particle Physics and Astrophysics (IPA), Zurich, Switzerland

M. Backhaus, L. Bäni, P. Berger, N. Chernyavskaya, G. Dissertori, M. Dittmar, M. Donegà, C. Dorfer, C. Grab, C. Heidegger, D. Hits, J. Hoss, T. Klijnsma, W. Lustermann, R.A. Manzoni, M. Marionneau, M.T. Meinhard, D. Meister, F. Micheli, P. Musella, F. Nessi-Tedaldi, J. Pata, F. Pauss, G. Perrin, L. Perrozzi, S. Pigazzini, M. Quittnat, M. Reichmann, D. Ruini, D.A. Sanz Becerra, M. Schönenberger, L. Shchutska, V.R. Tavolaro, K. Theofilatos, M.L. Vesterbacka Olsson, R. Wallny, D.H. Zhu

Universität Zürich, Zurich, Switzerland

T.K. Aarrestad, C. Amsler ${ }^{46}$, D. Brzhechko, M.F. Canelli, A. De Cosa, R. Del Burgo, S. Donato, C. Galloni, T. Hreus, B. Kilminster, I. Neutelings, D. Pinna, G. Rauco, P. Robmann, D. Salerno, K. Schweiger, C. Seitz, Y. Takahashi, A. Zucchetta

National Central University, Chung-Li, Taiwan

Y.H. Chang, K.y. Cheng, T.H. Doan, Sh. Jain, R. Khurana, C.M. Kuo, W. Lin, A. Pozdnyakov, S.S. Yu

National Taiwan University (NTU), Taipei, Taiwan

P. Chang, Y. Chao, K.F. Chen, P.H. Chen, W.-S. Hou, Arun Kumar, Y.y. Li, R.-S. Lu, E. Paganis, A. Psallidas, A. Steen, J.f. Tsai

Chulalongkorn University, Faculty of Science, Department of Physics, Bangkok, Thailand

B. Asavapibhop, N. Srimanobhas, N. Suwonjandee

Çukurova University, Physics Department, Science and Art Faculty, Adana, Turkey

A. Bat, F. Boran, S. Cerci ${ }^{47}$, S. Damarseckin, Z.S. Demiroglu, F. Dolek, C. Dozen, I. Dumanoglu, S. Girgis, G. Gokbulut, Y. Guler, E. Gurpinar, I. $\operatorname{Hos}^{48}$, C. Isik, E.E. Kangal ${ }^{49}$, O. Kara, A. Kayis Topaksu, U. Kiminsu, M. Oglakci, G. Onengut, K. Ozdemir ${ }^{50}$, S. Ozturk ${ }^{51}$, B. Tali ${ }^{47}$, U.G. Tok, H. Topakli ${ }^{51}$, S. Turkcapar, I.S. Zorbakir, C. Zorbilmez

Middle East Technical University, Physics Department, Ankara, Turkey

B. Isildak ${ }^{52}$, G. Karapinar ${ }^{53}$, M. Yalvac, M. Zeyrek

Bogazici University, Istanbul, Turkey

I.O. Atakisi, E. Gülmez, M. Kaya ${ }^{54}$, O. Kaya ${ }^{55}$, S. Ozkorucuklu ${ }^{56}$, S. Tekten, E.A. Yetkin ${ }^{57}$

Istanbul Technical University, Istanbul, Turkey

M.N. Agaras, S. Atay, A. Cakir, K. Cankocak, Y. Komurcu, S. Sen ${ }^{58}$

Institute for Scintillation Materials of National Academy of Science of Ukraine, Kharkov, Ukraine

B. Grynyov

National Scientific Center, Kharkov Institute of Physics and Technology, Kharkov, Ukraine

L. Levchuk 


\section{University of Bristol, Bristol, United Kingdom}

F. Ball, L. Beck, J.J. Brooke, D. Burns, E. Clement, D. Cussans, O. Davignon, H. Flacher, J. Goldstein, G.P. Heath, H.F. Heath, L. Kreczko, D.M. Newbold ${ }^{59}$, S. Paramesvaran, B. Penning, T. Sakuma, D. Smith, V.J. Smith, J. Taylor, A. Titterton

\section{Rutherford Appleton Laboratory, Didcot, United Kingdom}

K.W. Bell, A. Belyaev ${ }^{60}$, C. Brew, R.M. Brown, D. Cieri, D.J.A. Cockerill, J.A. Coughlan, K. Harder, S. Harper, J. Linacre, E. Olaiya, D. Petyt, C.H. Shepherd-Themistocleous, A. Thea, I.R. Tomalin, T. Williams, W.J. Womersley

\section{Imperial College, London, United Kingdom}

G. Auzinger, R. Bainbridge, P. Bloch, J. Borg, S. Breeze, O. Buchmuller, A. Bundock, S. Casasso, D. Colling, L. Corpe, P. Dauncey, G. Davies, M. Della Negra, R. Di Maria, Y. Haddad, G. Hall, G. Iles, T. James, M. Komm, C. Laner, L. Lyons, A.-M. Magnan, S. Malik, A. Martelli, J. Nash ${ }^{61}$, A. Nikitenko ${ }^{6}$, V. Palladino, M. Pesaresi, A. Richards, A. Rose, E. Scott, C. Seez, A. Shtipliyski, G. Singh, M. Stoye, T. Strebler, S. Summers, A. Tapper, K. Uchida, T. Virdee ${ }^{14}$, N. Wardle, D. Winterbottom, J. Wright, S.C. Zenz

\section{Brunel University, Uxbridge, United Kingdom}

J.E. Cole, P.R. Hobson, A. Khan, P. Kyberd, C.K. Mackay, A. Morton, I.D. Reid, L. Teodorescu, S. Zahid

\section{Baylor University, Waco, U.S.A.}

K. Call, J. Dittmann, K. Hatakeyama, H. Liu, C. Madrid, B. Mcmaster, N. Pastika, C. Smith

\section{Catholic University of America, Washington DC, U.S.A.}

R. Bartek, A. Dominguez

The University of Alabama, Tuscaloosa, U.S.A.

A. Buccilli, S.I. Cooper, C. Henderson, P. Rumerio, C. West

\section{Boston University, Boston, U.S.A.}

D. Arcaro, T. Bose, D. Gastler, D. Rankin, C. Richardson, J. Rohlf, L. Sulak, D. Zou

Brown University, Providence, U.S.A.

G. Benelli, X. Coubez, D. Cutts, M. Hadley, J. Hakala, U. Heintz, J.M. Hogan ${ }^{62}$, K.H.M. Kwok, E. Laird, G. Landsberg, J. Lee, Z. Mao, M. Narain, J. Pazzini, S. Piperov, S. Sagir ${ }^{63}$, R. Syarif, D. Yu

\section{University of California, Davis, Davis, U.S.A.}

R. Band, C. Brainerd, R. Breedon, D. Burns, M. Calderon De La Barca Sanchez, M. Chertok, J. Conway, R. Conway, P.T. Cox, R. Erbacher, C. Flores, G. Funk, W. Ko, O. Kukral, R. Lander, C. Mclean, M. Mulhearn, D. Pellett, J. Pilot, S. Shalhout, M. Shi, D. Stolp, D. Taylor, K. Tos, M. Tripathi, Z. Wang, F. Zhang

\section{University of California, Los Angeles, U.S.A.}

M. Bachtis, C. Bravo, R. Cousins, A. Dasgupta, A. Florent, J. Hauser, M. Ignatenko, N. Mccoll, S. Regnard, D. Saltzberg, C. Schnaible, V. Valuev 
University of California, Riverside, Riverside, U.S.A.

E. Bouvier, K. Burt, R. Clare, J.W. Gary, S.M.A. Ghiasi Shirazi, G. Hanson, G. Karapostoli, E. Kennedy, F. Lacroix, O.R. Long, M. Olmedo Negrete, M.I. Paneva, W. Si, L. Wang, H. Wei, S. Wimpenny, B.R. Yates

University of California, San Diego, La Jolla, U.S.A.

J.G. Branson, S. Cittolin, M. Derdzinski, R. Gerosa, D. Gilbert, B. Hashemi, A. Holzner, D. Klein, G. Kole, V. Krutelyov, J. Letts, M. Masciovecchio, D. Olivito, S. Padhi, M. Pieri, M. Sani, V. Sharma, S. Simon, M. Tadel, A. Vartak, S. Wasserbaech ${ }^{64}$, J. Wood, F. Würthwein, A. Yagil, G. Zevi Della Porta

University of California, Santa Barbara - Department of Physics, Santa Barbara, U.S.A.

N. Amin, R. Bhandari, J. Bradmiller-Feld, C. Campagnari, M. Citron, A. Dishaw, V. Dutta, M. Franco Sevilla, L. Gouskos, R. Heller, J. Incandela, A. Ovcharova, H. Qu, J. Richman, D. Stuart, I. Suarez, S. Wang, J. Yoo

California Institute of Technology, Pasadena, U.S.A.

D. Anderson, A. Bornheim, J.M. Lawhorn, H.B. Newman, T.Q. Nguyen, M. Spiropulu, J.R. Vlimant, R. Wilkinson, S. Xie, Z. Zhang, R.Y. Zhu

Carnegie Mellon University, Pittsburgh, U.S.A.

M.B. Andrews, T. Ferguson, T. Mudholkar, M. Paulini, M. Sun, I. Vorobiev, M. Weinberg

University of Colorado Boulder, Boulder, U.S.A.

J.P. Cumalat, W.T. Ford, F. Jensen, A. Johnson, M. Krohn, S. Leontsinis, E. MacDonald, T. Mulholland, K. Stenson, K.A. Ulmer, S.R. Wagner

Cornell University, Ithaca, U.S.A.

J. Alexander, J. Chaves, Y. Cheng, J. Chu, A. Datta, K. Mcdermott, N. Mirman, J.R. Patterson, D. Quach, A. Rinkevicius, A. Ryd, L. Skinnari, L. Soffi, S.M. Tan, Z. Tao, J. Thom, J. Tucker, P. Wittich, M. Zientek

Fermi National Accelerator Laboratory, Batavia, U.S.A.

S. Abdullin, M. Albrow, M. Alyari, G. Apollinari, A. Apresyan, A. Apyan, S. Banerjee, L.A.T. Bauerdick, A. Beretvas, J. Berryhill, P.C. Bhat, G. Bolla ${ }^{\dagger}$, K. Burkett, J.N. Butler, A. Canepa, G.B. Cerati, H.W.K. Cheung, F. Chlebana, M. Cremonesi, J. Duarte, V.D. Elvira, J. Freeman, Z. Gecse, E. Gottschalk, L. Gray, D. Green, S. Grünendahl, O. Gutsche, J. Hanlon, R.M. Harris, S. Hasegawa, J. Hirschauer, Z. Hu, B. Jayatilaka, S. Jindariani, M. Johnson, U. Joshi, B. Klima, M.J. Kortelainen, B. Kreis, S. Lammel, D. Lincoln, R. Lipton, M. Liu, T. Liu, J. Lykken, K. Maeshima, J.M. Marraffino, D. Mason, P. McBride, P. Merkel, S. Mrenna, S. Nahn, V. O'Dell, K. Pedro, C. Pena, O. Prokofyev, G. Rakness, L. Ristori, A. Savoy-Navarro ${ }^{65}$, B. Schneider, E. Sexton-Kennedy, A. Soha, W.J. Spalding, L. Spiegel, S. Stoynev, J. Strait, N. Strobbe, L. Taylor, S. Tkaczyk, N.V. Tran, L. Uplegger, E.W. Vaandering, C. Vernieri, M. Verzocchi, R. Vidal, M. Wang, H.A. Weber, A. Whitbeck 
University of Florida, Gainesville, U.S.A.

D. Acosta, P. Avery, P. Bortignon, D. Bourilkov, A. Brinkerhoff, L. Cadamuro, A. Carnes, M. Carver, D. Curry, R.D. Field, S.V. Gleyzer, B.M. Joshi, J. Konigsberg, A. Korytov, P. Ma, K. Matchev, H. Mei, G. Mitselmakher, K. Shi, D. Sperka, J. Wang, S. Wang

Florida International University, Miami, U.S.A.

Y.R. Joshi, S. Linn

Florida State University, Tallahassee, U.S.A.

A. Ackert, T. Adams, A. Askew, S. Hagopian, V. Hagopian, K.F. Johnson, T. Kolberg, G. Martinez, T. Perry, H. Prosper, A. Saha, A. Santra, V. Sharma, R. Yohay

Florida Institute of Technology, Melbourne, U.S.A.

M.M. Baarmand, V. Bhopatkar, S. Colafranceschi, M. Hohlmann, D. Noonan, M. Rahmani, T. Roy, F. Yumiceva

University of Illinois at Chicago (UIC), Chicago, U.S.A.

M.R. Adams, L. Apanasevich, D. Berry, R.R. Betts, R. Cavanaugh, X. Chen, S. Dittmer, O. Evdokimov, C.E. Gerber, D.A. Hangal, D.J. Hofman, K. Jung, J. Kamin, C. Mills, I.D. Sandoval Gonzalez, M.B. Tonjes, N. Varelas, H. Wang, X. Wang, Z. Wu, J. Zhang

The University of Iowa, Iowa City, U.S.A.

M. Alhusseini, B. Bilki ${ }^{66}$, W. Clarida, K. Dilsiz ${ }^{67}$, S. Durgut, R.P. Gandrajula, M. Haytmyradov, V. Khristenko, J.-P. Merlo, A. Mestvirishvili, A. Moeller, J. Nachtman, H. Ogul ${ }^{68}$, Y. Onel, F. Ozok ${ }^{69}$, A. Penzo, C. Snyder, E. Tiras, J. Wetzel

Johns Hopkins University, Baltimore, U.S.A.

B. Blumenfeld, A. Cocoros, N. Eminizer, D. Fehling, L. Feng, A.V. Gritsan, W.T. Hung, P. Maksimovic, J. Roskes, U. Sarica, M. Swartz, M. Xiao, C. You

The University of Kansas, Lawrence, U.S.A.

A. Al-bataineh, P. Baringer, A. Bean, S. Boren, J. Bowen, J. Castle, S. Khalil, A. Kropivnitskaya, D. Majumder, W. Mcbrayer, M. Murray, C. Rogan, S. Sanders, E. Schmitz, J.D. Tapia Takaki, Q. Wang

Kansas State University, Manhattan, U.S.A.

A. Ivanov, K. Kaadze, D. Kim, Y. Maravin, D.R. Mendis, T. Mitchell, A. Modak, A. Mohammadi, L.K. Saini, N. Skhirtladze

Lawrence Livermore National Laboratory, Livermore, U.S.A.

F. Rebassoo, D. Wright

University of Maryland, College Park, U.S.A.

A. Baden, O. Baron, A. Belloni, S.C. Eno, Y. Feng, C. Ferraioli, N.J. Hadley, S. Jabeen, G.Y. Jeng, R.G. Kellogg, J. Kunkle, A.C. Mignerey, F. Ricci-Tam, Y.H. Shin, A. Skuja, S.C. Tonwar, K. Wong 
Massachusetts Institute of Technology, Cambridge, U.S.A.

D. Abercrombie, B. Allen, V. Azzolini, A. Baty, G. Bauer, R. Bi, S. Brandt, W. Busza, I.A. Cali, M. D'Alfonso, Z. Demiragli, G. Gomez Ceballos, M. Goncharov, P. Harris, D. Hsu, M. Hu, Y. Iiyama, G.M. Innocenti, M. Klute, D. Kovalskyi, Y.-J. Lee, P.D. Luckey, B. Maier, A.C. Marini, C. Mcginn, C. Mironov, S. Narayanan, X. Niu, C. Paus, C. Roland, G. Roland, G.S.F. Stephans, K. Sumorok, K. Tatar, D. Velicanu, J. Wang, T.W. Wang, B. Wyslouch, S. Zhaozhong

University of Minnesota, Minneapolis, U.S.A.

A.C. Benvenuti, R.M. Chatterjee, A. Evans, P. Hansen, S. Kalafut, Y. Kubota, Z. Lesko, J. Mans, S. Nourbakhsh, N. Ruckstuhl, R. Rusack, J. Turkewitz, M.A. Wadud

University of Mississippi, Oxford, U.S.A.

J.G. Acosta, S. Oliveros

University of Nebraska-Lincoln, Lincoln, U.S.A.

E. Avdeeva, K. Bloom, D.R. Claes, C. Fangmeier, F. Golf, R. Gonzalez Suarez, R. Kamalieddin, I. Kravchenko, J. Monroy, J.E. Siado, G.R. Snow, B. Stieger

State University of New York at Buffalo, Buffalo, U.S.A.

A. Godshalk, C. Harrington, I. Iashvili, A. Kharchilava, D. Nguyen, A. Parker, S. Rappoccio, B. Roozbahani

Northeastern University, Boston, U.S.A.

G. Alverson, E. Barberis, C. Freer, A. Hortiangtham, D.M. Morse, T. Orimoto, R. Teixeira De Lima, T. Wamorkar, B. Wang, A. Wisecarver, D. Wood

Northwestern University, Evanston, U.S.A.

S. Bhattacharya, O. Charaf, K.A. Hahn, N. Mucia, N. Odell, M.H. Schmitt, K. Sung, M. Trovato, M. Velasco

University of Notre Dame, Notre Dame, U.S.A.

R. Bucci, N. Dev, M. Hildreth, K. Hurtado Anampa, C. Jessop, D.J. Karmgard, N. Kellams, K. Lannon, W. Li, N. Loukas, N. Marinelli, F. Meng, C. Mueller, Y. Musienko ${ }^{32}$, M. Planer, A. Reinsvold, R. Ruchti, P. Siddireddy, G. Smith, S. Taroni, M. Wayne, A. Wightman, M. Wolf, A. Woodard

The Ohio State University, Columbus, U.S.A.

J. Alimena, L. Antonelli, B. Bylsma, L.S. Durkin, S. Flowers, B. Francis, A. Hart, C. Hill, W. Ji, T.Y. Ling, W. Luo, B.L. Winer, H.W. Wulsin

Princeton University, Princeton, U.S.A.

S. Cooperstein, P. Elmer, J. Hardenbrook, P. Hebda, S. Higginbotham, A. Kalogeropoulos, D. Lange, M.T. Lucchini, J. Luo, D. Marlow, K. Mei, I. Ojalvo, J. Olsen, C. Palmer, P. Piroué, J. Salfeld-Nebgen, D. Stickland, C. Tully

University of Puerto Rico, Mayaguez, U.S.A.

S. Malik, S. Norberg 
Purdue University, West Lafayette, U.S.A.

A. Barker, V.E. Barnes, S. Das, L. Gutay, M. Jones, A.W. Jung, A. Khatiwada, B. Mahakud, D.H. Miller, N. Neumeister, C.C. Peng, H. Qiu, J.F. Schulte, J. Sun, F. Wang, R. Xiao, W. Xie

Purdue University Northwest, Hammond, U.S.A.

T. Cheng, J. Dolen, N. Parashar

Rice University, Houston, U.S.A.

Z. Chen, K.M. Ecklund, S. Freed, F.J.M. Geurts, M. Guilbaud, M. Kilpatrick, W. Li, B. Michlin, B.P. Padley, J. Roberts, J. Rorie, W. Shi, Z. Tu, J. Zabel, A. Zhang

University of Rochester, Rochester, U.S.A.

A. Bodek, P. de Barbaro, R. Demina, Y.t. Duh, J.L. Dulemba, C. Fallon, T. Ferbel, M. Galanti, A. Garcia-Bellido, J. Han, O. Hindrichs, A. Khukhunaishvili, K.H. Lo, P. Tan, R. Taus, M. Verzetti

Rutgers, The State University of New Jersey, Piscataway, U.S.A.

A. Agapitos, J.P. Chou, Y. Gershtein, T.A. Gómez Espinosa, E. Halkiadakis, M. Heindl, E. Hughes, S. Kaplan, R. Kunnawalkam Elayavalli, S. Kyriacou, A. Lath, R. Montalvo, K. Nash, M. Osherson, H. Saka, S. Salur, S. Schnetzer, D. Sheffield, S. Somalwar, R. Stone, S. Thomas, P. Thomassen, M. Walker

University of Tennessee, Knoxville, U.S.A.

A.G. Delannoy, J. Heideman, G. Riley, K. Rose, S. Spanier, K. Thapa

Texas A\&M University, College Station, U.S.A.

O. Bouhali ${ }^{70}$, A. Castaneda Hernandez ${ }^{70}$, A. Celik, M. Dalchenko, M. De Mattia, A. Delgado, S. Dildick, R. Eusebi, J. Gilmore, T. Huang, T. Kamon ${ }^{71}$, S. Luo, R. Mueller, Y. Pakhotin, R. Patel, A. Perloff, L. Perniè, D. Rathjens, A. Safonov, A. Tatarinov

Texas Tech University, Lubbock, U.S.A.

N. Akchurin, J. Damgov, F. De Guio, P.R. Dudero, S. Kunori, K. Lamichhane, S.W. Lee, T. Mengke, S. Muthumuni, T. Peltola, S. Undleeb, I. Volobouev, Z. Wang

Vanderbilt University, Nashville, U.S.A.

S. Greene, A. Gurrola, R. Janjam, W. Johns, C. Maguire, A. Melo, H. Ni, K. Padeken, J.D. Ruiz Alvarez, P. Sheldon, S. Tuo, J. Velkovska, M. Verweij, Q. Xu

\section{University of Virginia, Charlottesville, U.S.A.}

M.W. Arenton, P. Barria, B. Cox, R. Hirosky, M. Joyce, A. Ledovskoy, H. Li, C. Neu, T. Sinthuprasith, Y. Wang, E. Wolfe, F. Xia

Wayne State University, Detroit, U.S.A.

R. Harr, P.E. Karchin, N. Poudyal, J. Sturdy, P. Thapa, S. Zaleski 


\section{University of Wisconsin - Madison, Madison, WI, U.S.A.}

M. Brodski, J. Buchanan, C. Caillol, D. Carlsmith, S. Dasu, L. Dodd, S. Duric, B. Gomber, M. Grothe, M. Herndon, A. Hervé, U. Hussain, P. Klabbers, A. Lanaro, A. Levine, K. Long, R. Loveless, T. Ruggles, A. Savin, N. Smith, W.H. Smith, N. Woods

\section{t: Deceased}

1: Also at Vienna University of Technology, Vienna, Austria

2: Also at IRFU, CEA, Université Paris-Saclay, Gif-sur-Yvette, France

3: Also at Universidade Estadual de Campinas, Campinas, Brazil

4: Also at Federal University of Rio Grande do Sul, Porto Alegre, Brazil

5: Also at Université Libre de Bruxelles, Bruxelles, Belgium

6: Also at Institute for Theoretical and Experimental Physics, Moscow, Russia

7: Also at Joint Institute for Nuclear Research, Dubna, Russia

8: Also at Cairo University, Cairo, Egypt

9: Also at Helwan University, Cairo, Egypt

10: Now at Zewail City of Science and Technology, Zewail, Egypt

11: Also at Department of Physics, King Abdulaziz University, Jeddah, Saudi Arabia

12: Also at Université de Haute Alsace, Mulhouse, France

13: Also at Skobeltsyn Institute of Nuclear Physics, Lomonosov Moscow State University, Moscow, Russia

14: Also at CERN, European Organization for Nuclear Research, Geneva, Switzerland

15: Also at RWTH Aachen University, III. Physikalisches Institut A, Aachen, Germany

16: Also at University of Hamburg, Hamburg, Germany

17: Also at Brandenburg University of Technology, Cottbus, Germany

18: Also at Institute of Nuclear Research ATOMKI, Debrecen, Hungary

19: Also at MTA-ELTE Lendület CMS Particle and Nuclear Physics Group, Eötvös Loránd University, Budapest, Hungary

20: Also at Institute of Physics, University of Debrecen, Debrecen, Hungary

21: Also at Indian Institute of Technology Bhubaneswar, Bhubaneswar, India

22: Also at Institute of Physics, Bhubaneswar, India

23: Also at Shoolini University, Solan, India

24: Also at University of Visva-Bharati, Santiniketan, India

25: Also at Isfahan University of Technology, Isfahan, Iran

26: Also at Plasma Physics Research Center, Science and Research Branch, Islamic Azad University, Tehran, Iran

27: Also at Università degli Studi di Siena, Siena, Italy

28: Also at International Islamic University of Malaysia, Kuala Lumpur, Malaysia

29: Also at Malaysian Nuclear Agency, MOSTI, Kajang, Malaysia

30: Also at Consejo Nacional de Ciencia y Tecnología, Mexico city, Mexico

31: Also at Warsaw University of Technology, Institute of Electronic Systems, Warsaw, Poland

32: Also at Institute for Nuclear Research, Moscow, Russia

33: Now at National Research Nuclear University 'Moscow Engineering Physics Institute' (MEPhI), Moscow, Russia

34: Also at St. Petersburg State Polytechnical University, St. Petersburg, Russia

35: Also at University of Florida, Gainesville, U.S.A.

36: Also at P.N. Lebedev Physical Institute, Moscow, Russia

37: Also at California Institute of Technology, Pasadena, U.S.A. 
38: Also at Budker Institute of Nuclear Physics, Novosibirsk, Russia

39: Also at Faculty of Physics, University of Belgrade, Belgrade, Serbia

40: Also at INFN Sezione di Pavia ${ }^{a}$, Università di Pavia ${ }^{b}$, Pavia, Italy

41: Also at University of Belgrade, Faculty of Physics and Vinca Institute of Nuclear Sciences, Belgrade, Serbia

42: Also at Scuola Normale e Sezione dell'INFN, Pisa, Italy

43: Also at National and Kapodistrian University of Athens, Athens, Greece

44: Also at Riga Technical University, Riga, Latvia

45: Also at Universität Zürich, Zurich, Switzerland

46: Also at Stefan Meyer Institute for Subatomic Physics (SMI), Vienna, Austria

47: Also at Adiyaman University, Adiyaman, Turkey

48: Also at Istanbul Aydin University, Istanbul, Turkey

49: Also at Mersin University, Mersin, Turkey

50: Also at Piri Reis University, Istanbul, Turkey

51: Also at Gaziosmanpasa University, Tokat, Turkey

52: Also at Ozyegin University, Istanbul, Turkey

53: Also at Izmir Institute of Technology, Izmir, Turkey

54: Also at Marmara University, Istanbul, Turkey

55: Also at Kafkas University, Kars, Turkey

56: Also at Istanbul University, Faculty of Science, Istanbul, Turkey

57: Also at Istanbul Bilgi University, Istanbul, Turkey

58: Also at Hacettepe University, Ankara, Turkey

59: Also at Rutherford Appleton Laboratory, Didcot, United Kingdom

60: Also at School of Physics and Astronomy, University of Southampton, Southampton, United Kingdom

61: Also at Monash University, Faculty of Science, Clayton, Australia

62: Also at Bethel University, St. Paul, U.S.A.

63: Also at Karamanoğlu Mehmetbey University, Karaman, Turkey

64: Also at Utah Valley University, Orem, U.S.A.

65: Also at Purdue University, West Lafayette, U.S.A.

66: Also at Beykent University, Istanbul, Turkey

67: Also at Bingol University, Bingol, Turkey

68: Also at Sinop University, Sinop, Turkey

69: Also at Mimar Sinan University, Istanbul, Istanbul, Turkey

70: Also at Texas A\&M University at Qatar, Doha, Qatar

71: Also at Kyungpook National University, Daegu, Korea 